\title{
Theory of out-of-equilibrium electron and phonon dynamics in metals after femtosecond laser excitation
}

\author{
Ulrike Ritzmann $\odot,,^{1,2,3}$ Peter M. Oppeneer $\odot,{ }^{1,2}$ and Pablo Maldonado ${ }^{1}$ \\ ${ }^{1}$ Department of Physics and Astronomy, Uppsala University, Box 516, S-75120 Uppsala, Sweden \\ ${ }^{2}$ Department of Physics, Freie Universität Berlin, Arnimallee 14, D-14195 Berlin, Germany \\ ${ }^{3}$ Dahlem Center of Complex Quantum Systems, Freie Universität Berlin, Arnimallee 14, D-14195 Berlin, Germany
}

(Received 27 November 2019; revised 10 November 2020; accepted 4 December 2020; published 18 December 2020)

\begin{abstract}
The dynamics of electrons and phonons in metals upon laser excitation are often described by the twotemperature model, which assumes that both subsystems are individually in thermal equilibrium. However, recent experiments show that this description is not sufficient to describe the out-of-equilibrium dynamics on ultrashort timescales. Here, assuming a thermalized electronic system, we extend and apply a parameter-free microscopic out-of-equilibrium model to describe the ultrafast laser-induced phonon and electron temperature dynamics of various metallic systems such as gold, aluminum, iron, nickel, and cobalt. We report strong deviations from the two-temperature model on the picosecond timescale for all the materials studied, even for those where the assumption of separate thermal equilibrium seemed less restrictive, like in gold. Furthermore, we demonstrate the importance of the mode dependence of the electron-phonon coupling for the relaxation process and reveal the significance of this channel in the lattice equilibration.
\end{abstract}

DOI: 10.1103/PhysRevB.102.214305

\section{INTRODUCTION}

The development of new experimental techniques using ultrashort laser pulses has allowed in recent years to gain access to novel phenomena taking place at subpicosecond timescales when the system is still strongly out-of-equilibrium [1-4] with heavily intertwined degrees of freedom. Thus, the complex interplay of electronic, phononic, and spin degrees of freedom after laser excitation has become the focus of contemporary research which has led to the discovery of new phenomena such as ultrafast demagnetization [5-7], change of magnetic anisotropy [8], ultrafast generation of lattice strain waves $[9,10]$, coherent phonon generation [11,12], laser-induced superconductivity at high temperatures [13], excitation of ultrafast spin currents [14] and of high-frequency exchange magnons [15], spintronic THz emitters [16], or femtosecond activation of magnetoelectricity [17].

From a theoretical perspective, thermal models such as the two- and three-temperature model are commonly used to describe the ultrafast interplay of electrons, phonons, and spins. These models are based on the assumption that the laser excites a thermalized electron distribution and that electrons and phonons are each individually in thermal equilibrium during the relaxation, and were originally derived by Kaganov et al. [18] and later extended by Anisimov et al. [19] for the case

Published by the American Physical Society under the terms of the Creative Commons Attribution 4.0 International license. Further distribution of this work must maintain attribution to the author(s) and the published article's title, journal citation, and DOI. Funded by Bibsam. of phonon and electron subsystems. The assumption implies that the electron system thermalizes instantaneously through electron-electron interaction and similarly, the phonon system thermalizes immediately through phonon-phonon interaction or that the electron-phonon coupling and specific heat is independent of the phonon modes. The time evolution of the two subsystems can then be described by the time dependence of the electron temperature $T_{\mathrm{e}}$ and the phonon temperature $T_{\mathrm{p}}$ derived from the following coupled equations [20]:

$$
\begin{gathered}
C_{\mathrm{e}} \frac{\partial T_{\mathrm{e}}^{2 \mathrm{TM}}}{\partial t}=-G_{\mathrm{ep}}\left(T_{\mathrm{p}}^{2 \mathrm{TM}}-T_{\mathrm{e}}^{2 \mathrm{TM}}\right)+P(t), \\
C_{\mathrm{p}} \frac{\partial T_{\mathrm{p}}^{2 \mathrm{TM}}}{\partial t}=G_{\mathrm{ep}}\left(T_{\mathrm{p}}^{2 \mathrm{TM}}-T_{\mathrm{e}}^{2 \mathrm{TM}}\right) .
\end{gathered}
$$

The specific heat constants for electrons $C_{\mathrm{e}}$ and phonons $C_{\mathrm{p}}$, as well as the electron-phonon coupling constant $G_{\text {ep }}$, can be determined either experimentally or by first-principles calculations [21]. $P(t)$ represents the absorbed power from the laser excitation.

However, recent experimental and theoretical works have evidenced that ultrashort laser pulses induce strong outof-equilibrium dynamics that cannot be described by simplified thermal models in the subpicosecond time regime $[10,22,23]$. As a consequence, a large number of theoretical and experimental studies have tried to shine light on the laser-induced out-of-equilibrium dynamics [24-35]. New theoretical models have been derived to improve the modeling on ultrashort timescales. One approach is to consider distinct phonon-branch dependent dynamics [22]. Further approaches introduce mode-dependent couplings and consider out-ofequilibrium distributions for electrons or phonons [36-40]. 
Here we use a microscopic out-of-equilibrium dynamics model derived by Maldonado et al. to investigate laserinduced dynamics of the phononic system in different metallic materials on picosecond timescales based on first-principles calculations [36]. The model includes phonon-mode dependent electron-phonon and phonon-phonon coupling. It is based on the notion that, in contrast to the phonon system, the electron system thermalizes very fast, by electron-electron scattering on the femtosecond timescale, leading to an approximately uniform electron temperature within 50 fs [41-44]. We discuss the out-of-equilibrium dynamics in gold, aluminum, nickel, iron, and cobalt. Our results reveal strong deviations from those provided by the two-temperature model even a long time after the laser excitation, evidencing thus the failure of the two-temperature model at these timescales. We demonstrate the relevance of the mode-dependent electronphonon coupling and show the distinct dynamical behavior of the different materials studied. Furthermore, we show that electron-phonon coupling has a significant role in the lattice equilibration mechanism, specifically, as a channel to transfer heat from hot to cold phonon modes via the electronic system.

\section{MICROSCOPIC OUT-OF-EQUILIBRIUM DYNAMICS MODEL}

In the following we will describe the theoretical model which provides the out-of-equilibrium system dynamics triggered by an ultrashort laser pulse in different metals, showing additionally the microscopic mechanisms involved in the equilibration process. Here it is important to mention that although the out-of-equilibrium electron dynamics is of special relevance to understand different laser-induced ultrafast phenomena, such as ultrafast demagnetization [5] or energy relaxation in strongly correlated electronic systems [45], it has been shown recently that the electronic relaxation time is only about a few tens of femtoseconds [41-44]. Considering that the relevant timescales of this work span several tens of picoseconds, we can safely assume a thermal distribution of the electronic system during the whole process with a transient electron temperature $T_{\mathrm{e}}(t)$. Contrarily, the lattice dynamics needs an explicit out-of-equilibrium representation of the phonon population [36].

To determine the change of the phononic distribution function, $\dot{n}_{Q}=\partial n_{Q} / \partial t$, it is important to consider different modifying processes such as diffusion, external fields, and scattering. Within the Boltzmann transport model they are usually represented as

$$
\dot{n}_{Q}=\dot{n}_{Q}^{\text {field }}+\dot{n}_{Q}^{\text {scatt. }}+\dot{n}_{Q}^{\text {dif. }},
$$

where $Q \equiv(\mathbf{q}, v)$ with $\mathbf{q}$ and $v$ being the phonon wave vector and branch, respectively. Assuming that the external field caused by the laser has disappeared at time $t=0$ and also considering only relatively short timescales after the laser excitation and a constrained geometry, we can neglect the influence of diffusion and external field on the change of the phonon population. Therefore, we only consider the scattering term as the driver of the relaxation between the laser excited electron bath and the phonons, and we can write

$$
\dot{n}_{Q}=\dot{n}_{Q}^{\text {scatt. }} .
$$

The scattering term can be determined employing many-body perturbation theory and Fermi's golden rule to estimate the probability of a transition between two states due to a specific scattering mechanism [46]. Since in this work we are only interested in the interactions among electrons and phonons, we have

$$
\dot{n}_{Q}=\dot{n}_{Q}^{\mathrm{e}-\mathrm{p}}+\dot{n}_{Q}^{\mathrm{p}-\mathrm{p}},
$$

where $\dot{n}_{Q}^{\mathrm{e}-\mathrm{p}}$ and $\dot{n}_{Q}^{\mathrm{p}-\mathrm{p}}$ denote the scattering terms due to electronphonon and phonon-phonon interaction [46], respectively, with

$$
\begin{aligned}
\dot{n}_{Q}^{\mathrm{p}-\mathrm{p}}=\frac{2 \pi}{\hbar^{2}} \frac{36}{2} \sum_{k, k^{\prime}}\left|\Phi_{-Q, k, k^{\prime}}\right|^{2}\left[\left(n_{Q}+1\right)\left(n_{k}+1\right) n_{k^{\prime}} \delta\left(\omega_{Q}+\omega_{k^{\prime}}-\omega_{k^{\prime \prime}}\right)+\left(n_{Q}+1\right)\left(n_{k^{\prime}}+1\right) n_{k} \delta\left(\omega_{Q}+\omega_{k^{\prime}}\right.\right. \\
\left.-\omega_{k}\right)-n_{Q} n_{k}\left(n_{k^{\prime}}+1\right) \delta\left(\omega_{Q}-\omega_{k^{\prime}}+\omega_{k}\right)-n_{Q} n_{k^{\prime}}\left(n_{k}+1\right) \delta\left(\omega_{Q}+\omega_{k^{\prime}}-\omega_{k}\right) \\
\left.+\left(n_{Q}+1\right) n_{k} n_{k^{\prime}} \delta\left(\omega_{Q}-\omega_{k^{\prime}}-\omega_{k}\right)-n_{Q}\left(n_{k}+1\right)\left(n_{k^{\prime}}+1\right) \delta\left(\omega_{Q}-\omega_{k^{\prime}}-\omega_{k}\right)\right] .
\end{aligned}
$$

Here $\omega_{Q}$ is the frequency of the phonon mode $Q$ and the $\Phi_{-Q, k, k^{\prime}}$ are the matrix elements that involve three-phonon scattering processes $[47,48]$, for details see Appendix A) and

$$
\begin{aligned}
\dot{n}_{Q}^{\mathrm{e}-\mathrm{p}}= & -\frac{4 \pi}{\hbar} \frac{1}{N} \sum_{k n n^{\prime} \sigma}\left|g_{n n^{\prime} \mathbf{k}^{\prime}}^{\sigma}(\mathbf{k}, \mathbf{q})\right|^{2} \delta\left(\epsilon_{\mathbf{k} n}^{\sigma}-\epsilon_{\mathbf{k}+\mathbf{q}, n^{\prime}}^{\sigma}+\hbar \omega_{Q}\right) \\
& \times\left[n_{Q} f_{n \mathbf{k}}^{\sigma}\left(1-f_{n^{\prime} \mathbf{k}^{\prime}}^{\sigma}\right)-f_{n^{\prime} \mathbf{k}^{\prime}}^{\sigma}\left(1-f_{n \mathbf{k}}^{\sigma}\right)\left(n_{Q}+1\right)\right],
\end{aligned}
$$

with $g_{n n^{\prime} \mathbf{k}^{\prime}}^{\sigma}(\mathbf{k}, \mathbf{q})$ being the electron-phonon matrix elements that couples the electronic states of different quantum numbers (k wave vector, $n$ band index) but with the same $\sigma$ spin through a phonon $Q . f_{n \mathbf{k}}^{\sigma}$ is the transient electronic population of the state specified by quantum numbers $n \mathbf{k}$ and $\sigma$. Under the assumption of a thermalized electronic system it becomes the Fermi-Dirac distribution with transient electronic temperature $T_{\mathrm{e}}(t)$, which can be obtained by solving

$$
\frac{\partial E_{\mathrm{e}}}{\partial t}=-\sum_{Q} \hbar \omega_{Q} \dot{n}_{Q}^{\mathrm{e}-\mathrm{p}}+P(t),
$$

with $P(t)$ the absorbed laser power.

Hence, we can determine the transient phonon dynamics by solving numerically the set of coupled equations provided by Eqs. (4), (6), (7), and (8), where the only unknown quantity in the description, which are the distinct electron-phonon matrix elements, can be computed from first-principles calculations (see Appendix B). 
Alternatively, we can use an equivalent formulation based on an "effective" phonon temperature following that the phonon distributions are continuous functions. In doing so, we use the ansatz that the phononic out-of-equilibrium distribution function can be written as

$$
n_{Q}(t)=\frac{1}{\exp \left(\frac{\hbar \omega_{Q}}{k_{\mathrm{B}} T_{\mathrm{p}}(t)}+\Psi_{Q}(t)\right)-1},
$$

where $T_{\mathrm{p}}(t)$ is the lattice temperature and $\Psi_{Q}$ is a measurement of the deviation from equilibrium [49,50]. Correspondingly, we can rewrite the above expression with a more compact notation by defining a phonon mode-dependent effective phonon temperature $T_{\mathrm{p}}{ }^{Q}(t)$ that can notably differ for distinct regions of the Brillouin zone (BZ),

$$
n_{Q}(t)=\frac{1}{\exp \left(\frac{\hbar \omega_{Q}}{k_{\mathrm{B}} T_{\mathrm{p}}^{Q}(t)}\right)-1} .
$$

These branch and wave-vector-dependent phonon temperatures have their counterparts for the electronic states at different regions of the Fermi surface as proposed theoretically by Schutt et al. [51]. Then, by using the conservation of total energy and the Boltzmann kinetic theory, a set of coupled equations of motion for the temperatures of each phonon mode $Q$ and of the electron bath can be derived:

$$
C_{\mathrm{e}} \frac{\partial T_{\mathrm{e}}}{\partial t}=\sum_{Q} \gamma_{Q} C_{Q}\left(T_{\mathrm{p}}^{Q}-T_{\mathrm{e}}\right) I\left[1+J\left(T_{\mathrm{p}}^{Q}-T_{\mathrm{e}}\right)\right]+P(t),
$$

$$
\begin{aligned}
C_{Q} \frac{\partial T_{\mathrm{p}}^{Q}}{\partial t}=-\gamma_{Q} C_{Q}\left(T_{\mathrm{p}}^{Q}-T_{\mathrm{e}}\right) & {\left[1+J\left(T_{\mathrm{p}}^{Q}-T_{\mathrm{e}}\right)\right] I\left(T_{e}\right) } \\
& -\sum_{k^{\prime}} C_{Q} \Gamma_{Q k^{\prime}}\left(T_{\mathrm{p}}^{Q}-\tilde{T}_{\mathrm{p}}^{k, k^{\prime}}\right) \\
\times & {\left[1+J\left(T_{\mathrm{p}}^{Q}-\tilde{T}_{\mathrm{p}}^{k, k^{\prime}}\right)\right], }
\end{aligned}
$$

where $C_{\mathrm{e}}$ and $C_{Q}$ are the electronic and phonon-mode $Q$ heat capacities, and $\gamma_{Q}$ and $\Gamma_{Q k^{\prime}}$ are the phonon linewidths due to electron-phonon and phonon-phonon scattering, respectively. Note than only electrons around the Fermi level have been taken into account, consistent with the energy interval achievable by the hot electron temperatures. The effect of high electronic temperatures has furthermore been included through the function $I\left(T_{e}\right)$. These quantities are obtained from first-principles calculations, in our case on a dense grid of $20^{3} k$ points. Details of the derivation of these equations can be found in Appendix A (see also Maldonado et al. [36]). However, it is important to mention that the model [36] has been further improved in this work to explicitly avoid the use of the single relaxation approximation in the derivation of Eqs. (11) and (12).

The laser excitation is considered to have a Gaussian distribution with the rate of the absorbed energy given by

$$
P(t)=(1-R) \phi \alpha \frac{2}{s \sqrt{2 \pi}} \exp \left(-\frac{-2 t^{2}}{s^{2}}\right) .
$$

The full width at half-maximum of the laser pulse $\sigma$ is $100 \mathrm{fs}$ and given by $\sigma=\sqrt{s /[2 \sqrt{2 \ln (2)}]}$. We consider a laser fluence of $\phi=50 \mathrm{~mJ} / \mathrm{cm}^{2} . R$ is the reflectivity and $\alpha$ is the adsorption coefficient, for which we use values at room temperature. For a better comparison of the results obtained for the different metallic systems here studied, we use the same rate of absorbed energy for all cases. The amount of absorbed energy is defined initially for gold, and then taken as a reference for the other systems. In this case, the reflectivity is given by $R=0.98$ and the absorption coefficient is $\alpha=0.80916$ $\mathrm{cm}^{-1}$ for a $800 \mathrm{~nm}$ wavelength of the laser pulse. Note that the adsorption coefficients can vary largely for the materials and therefore the laser fluences have been modified accordingly.

In the following we will use the "effective" phonon temperature formulation, and solve Eqs. (11) and (12) rather than obtaining the out-of-equilibrium phonon populations directly from Eqs. (4), (6), (7), and (8), to have a transparent and direct way to check the validity of the two-temperature model and to establish a clear framework for those investigations that use the electron, lattice, or spin transient temperatures to model different ultrafast physical phenomena [48,52-57]. Additionally, this formulation allows for an easy interpretation of the energy flow, able to account for different "hot" and "cold" dissipation channels, e.g., in highly correlated systems [45]. Finally, it is worth mentioning that this formulation is equivalent to solving directly the rate equations in the form of phonon populations [Eqs. (4), (6), (7), and (8)] up to second order in the effective phonon temperature expansion and for the low fluences used in this work (see Appendix A). Thus, the here-developed formalism can equally well be used to provide the transient phonon dynamics from which we can compute relevant experimental quantities such as the diffusive scattering and Bragg diffraction intensities $[39,45,58]$.

\section{RESULTS}

Before analyzing the out-of-equilibrium dynamics obtained as a solution of Eqs. (11) and (12) for the different metallic systems here studied, i.e., gold, aluminum, iron nickel, and cobalt, we have computed the phonon-mode dependent linewidths using the ABINIT software [59] to extract the electron-phonon couplings and the system's force constants. We have also calculated the electron-phonon coupling constant $G_{\text {ep }}=\sum_{Q} \gamma_{Q} C_{Q}$, commonly used as a free parameter in the two-temperature model. In our case $G_{\text {ep }}$ will be used to compare the results of our out-of-equilibrium model with the two-temperature model. The results for the different materials computed at $T=300 \mathrm{~K}$ are summarized in Table I, along with averaged values for the phonon linewidth due to electron-phonon coupling $\left\langle\gamma_{Q}\right\rangle=1 / N_{Q} \sum_{Q} \gamma_{Q}$, and the averaged value for the phonon linewidth due to phonon-phonon coupling $\left\langle\Gamma_{Q}\right\rangle=1 / N_{Q} \sum_{Q} \Gamma_{Q}$.

Importantly, we obtain that in gold and aluminum the role of anharmonicities is more significant, indicated by the larger phonon-phonon linewidth as compared to nickel, iron, and cobalt. Moreover, in the case of gold this coupling is even larger than the electron-phonon coupling strength, and therefore a fast lattice equilibration could be expected, which would justify the use of the two-temperature model. Contrarily, we find for the rest of the systems studied that the 
TABLE I. Computed material's specific constants. Given are the averaged linewidth for electron-phonon coupling $\left\langle\gamma_{Q}\right\rangle$, and for phonon-phonon coupling $\left\langle\Gamma_{Q}\right\rangle$, and the corresponding electronphonon coupling constant $G_{\text {ep }}$.

\begin{tabular}{lccc}
\hline \hline Material & $\left\langle\gamma_{Q}\right\rangle(\mathrm{GHz})$ & $\left\langle\Gamma_{Q}\right\rangle(\mathrm{GHz})$ & $G_{\text {ep }}\left[10^{17} \mathrm{~W} /\left(\mathrm{m}^{3} \mathrm{~K}\right)\right]$ \\
\hline Gold & 4.7 & 90.6 & 0.225 \\
Aluminum & 101.1 & 77.2 & 4.47 \\
Iron & 164.0 & 6.7 & 10.5 \\
Nickel & 272.5 & 27.0 & 18.9 \\
Cobalt & 458.7 & 16.8 & 33.4 \\
\hline \hline
\end{tabular}

electron-phonon strength coupling is larger than the phononphonon coupling strength, and therefore it could be already expected that the lattice cannot be modeled with a thermal distribution having a single lattice temperature. The values of the electron-phonon coupling constants reported in Table I are in good agreement with previously published works, except for the case of iron where a large deviation is observed [60]. In contrast to our work, where we use first-principles computation of the quantities, the electron-phonon coupling constant was previously estimated by using a Debye approximation, to which we associate the discrepancies with our results.

Here it is important to stress that our model has been derived under the assumption that the spin subsystem plays a negligible role in the lattice dynamics due to the usually small magnetic heat capacities that lead to a small energy absorption in the spin systems. Although this assumption is rigorously valid for nonmagnetic materials such as $\mathrm{Au}$ and $\mathrm{Al}$ it is only valid at low temperatures for magnetic systems and it might fail close to the Curie temperature. On the other hand, we include an explicit dependence of the electron-phonon coupling on the electron spins (the total electron-phonon coupling is the result of the sum of the coupling for minority and majority electrons). Here we consider the spin-dependent values for fully magnetized systems. Although we assume that the inclusion of the spin subsystem in our model will modify the quantitative behavior of the dynamics, the qualitative behavior would be the same, and therefore the timescales of the dynamics and conclusions drawn in this work remain valid.

\section{A. Out-of-equilibrium dynamics in gold}

One of the main assumptions of the two-temperature model is that phonons are in complete thermal equilibrium during the interaction with the electrons. This condition can be fulfilled either by assuming an homogeneous electron-phonon coupling such that the heat is homogeneously distributed or by assuming a phonon-phonon interaction strength much larger than the electron-phonon coupling strength such that the lattice is equilibrated instantaneously. As for the case of $\mathrm{Au}$, the former condition is not fulfilled as we can see in Fig. 1(a) where we show the calculated mode-dependent phonon linewidth due to electron-phonon interaction as a function of the frequency. Note that multiple points at one frequency occur, since each data point represents a phonon at the state $(\nu \mathbf{k})$. The broad dispersion of linewidths along the phonon frequencies evidences how the energy will be largely inhomogeneously distributed among the different phonon
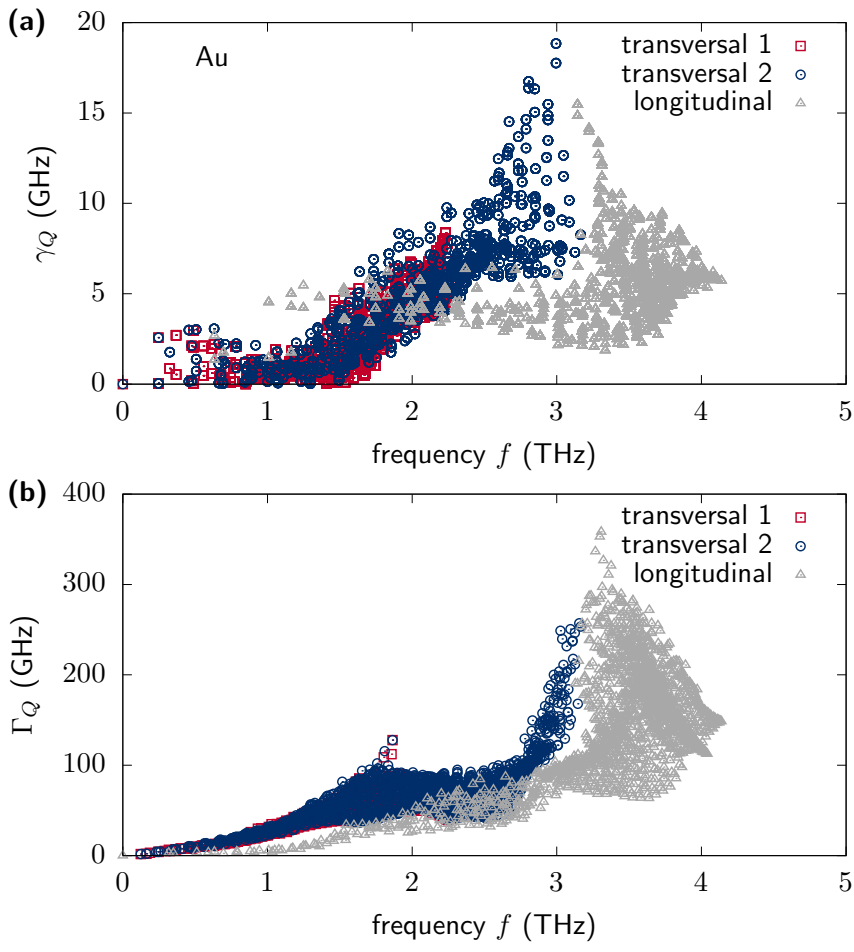

FIG. 1. Calculated electron-phonon linewidths (top) and phonon-phonon linewidths (bottom) in gold as a function of the phonon frequency for the three phonon branches.

modes. On the other hand, the phonon linewidths due to the phonon-phonon interaction, which are also strongly mode dependent, present values that are one order of magnitude larger than those stemming from the electron-phonon scatterings, as illustrated in Fig. 1(b). This potentially suggest the validity of a thermal description of the lattice in the two-temperature model for Au. Quantitatively this is also suggested by the averaged electron-phonon linewidth which has a value of about $4.7 \mathrm{GHz}$, corresponding to an averaged lifetime of $106 \mathrm{ps}$, whereas the averaged phonon-phonon linewidth is $90.6 \mathrm{GHz}$ corresponding to a lifetime of $5.5 \mathrm{ps}$.

We compute the dynamics of the electron and phonon systems that are initially at $300 \mathrm{~K}$ after laser excitation by using the two-temperature model, given by Eqs. (1) and (2), as well as by the microscopic out-of-equilibrium dynamics model described by Eqs. (11) and (12). To compare the two models, we calculate the averaged temperature $T_{\mathrm{p}}^{\text {avg }}$, as well as the minimum and maximum effective temperature of the phonons $T_{\mathrm{p}}^{\min }$ and $T_{\mathrm{p}}^{\max }$. The results are shown in Fig. 2(a). The dynamics of the electron temperature $T_{\mathrm{e}}$ and the averaged effective phonon temperature $T_{\mathrm{p}}^{\text {avg }}$ of the microscopic out-of-equilibrium dynamics model are very similar to the predictions for the electron temperature $T_{\mathrm{e}}^{2 \mathrm{TM}}$ and the phonon temperature $T_{\mathrm{p}}^{2 \mathrm{TM}}$ from the two-temperature model when using our computed parameters. The electron subsystem is heated up to almost $2400 \mathrm{~K}$ within the first picosecond and afterwards the system equilibrates within about 12 ps having a final temperature of about $380 \mathrm{~K}$. The electron and lattice temperature evolution in the microscopic out-of-equilibrium dynamics model are slightly faster than in the two-temperature model. 

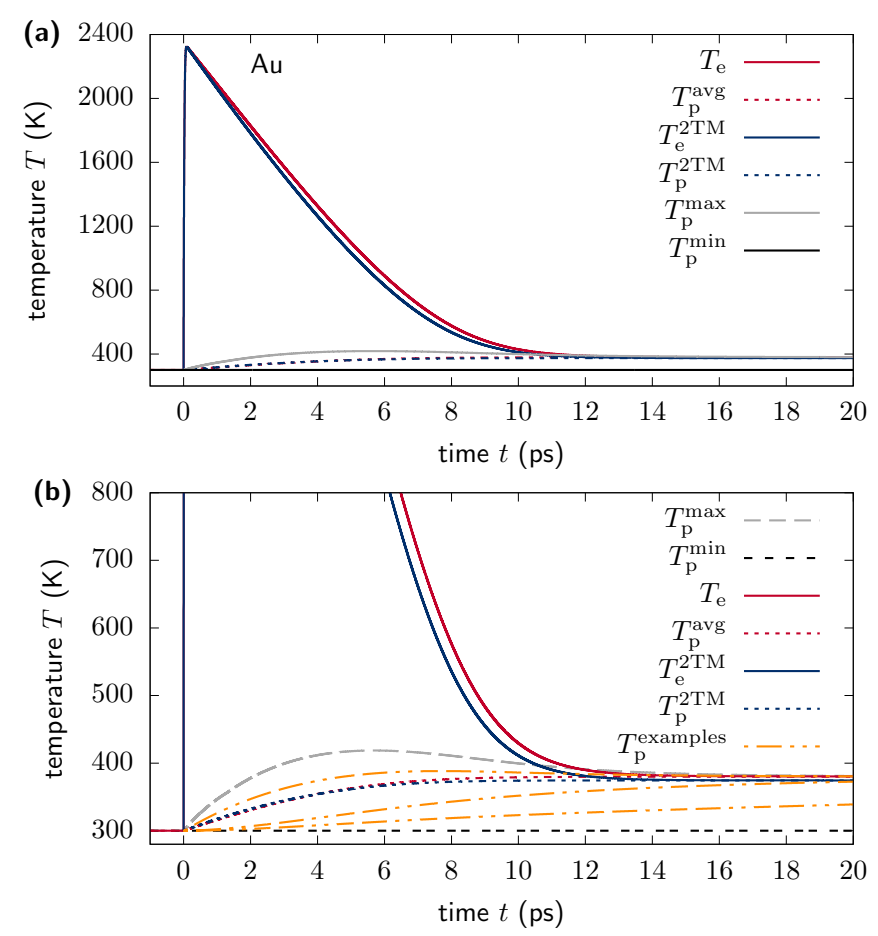

FIG. 2. Time evolution of the electron and phonon temperatures in gold. (a) Comparison of the temperatures computed from the two-temperature model (labeled 2TM) with those of the microscopic out-of-equilibrium dynamics model as a function of time after laser excitation. (b) Time dependence of exemplary phonon modes illustrating their different relaxation dynamics.

Furthermore, the large inhomogeneous energy distribution due to the strongly mode-dependent electron-phonon coupling is reflected in the very different dynamics of the maximum, minimum, and averaged effective temperatures. On the one hand, the maximum effective phonon temperature reaches a value of about $415 \mathrm{~K}$ at $\approx 5$ ps while the averaged effective phonon temperature only slowly converges to $380 \mathrm{~K}$ at around $10 \mathrm{ps}$. On the other hand, a few modes remain with temperatures around $300 \mathrm{~K}$ after $20 \mathrm{ps}$, since they couple very weakly or do not couple with electrons nor with other phonons, but possibly only to impurities which are not considered in our model. In Fig. 2(b) we show the temporal evolution of the effective temperatures of exemplary phonon modes by several dashed-dotted orange lines. Here we have chosen two modes with low energy and weak electron-phonon coupling and one phonon mode with high energy and a large electron-phonon coupling. These examples demonstrate that the temporal evolution of individual modes can differ significantly from the averaged time evolution. Furthermore, we observe in Fig. 2(b) that the maximum effective phonon temperature can even exceed the electron temperature, which is a clear evidence of the lattice being nonthermal. Further details on the modedependent effective phonon temperature distribution can be found in Appendix C, where we show that phonon modes with large electron-phonon coupling strength, corresponding to high energy phonons, reach higher effective temperatures, while low energy phonon modes-which couple weakly to electrons and other phonons-correspond to phonons with

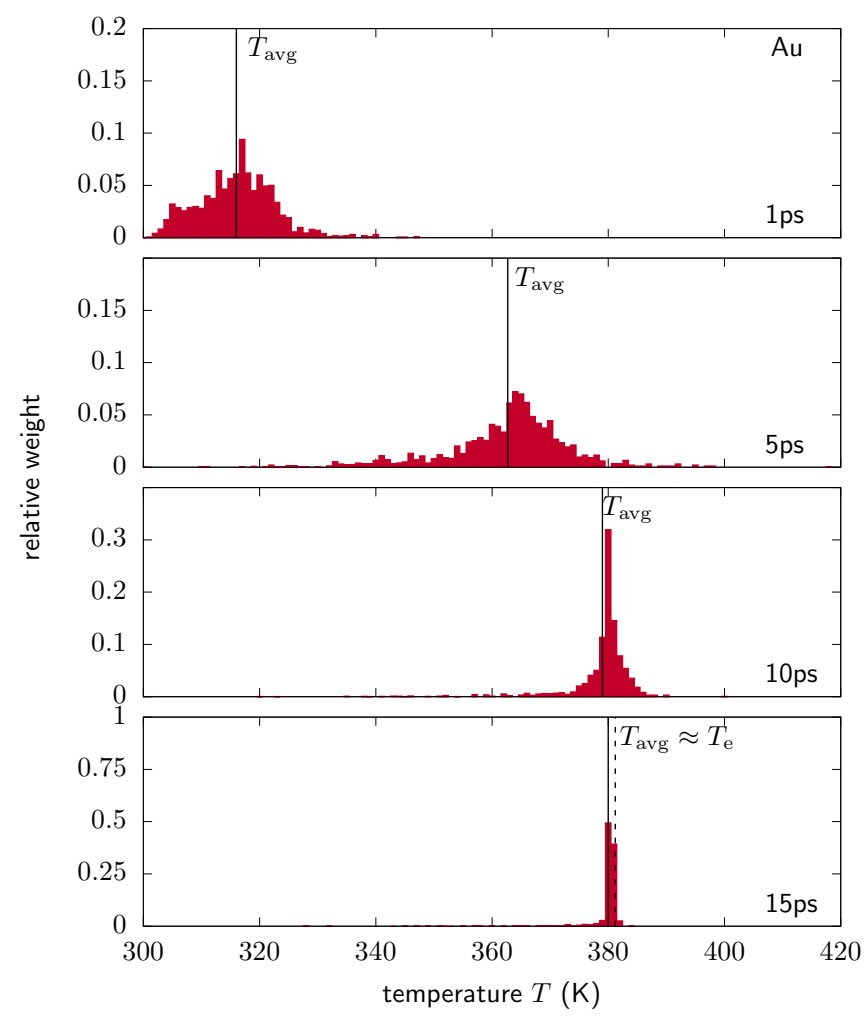

FIG. 3. Phononic temperature distribution at different times during the electron-phonon relaxation in gold. The red bars show the computed relative weights of phonon frequencies having a temperature within a range of $1 \mathrm{~K}$ for several different times. Vertical black lines give the average phonon temperature $T_{\text {avg }}$ and the vertical dashed line gives the electron temperature $T_{\mathrm{e}}$.

low effective temperatures that thermalize slowly and are not thermalized even at $10 \mathrm{ps}$ after laser pumping.

We gain further details of the out-of-equilibrium system dynamics by plotting in Fig. 3 the relative weight of all the phonon modes at a specific temperature within an interval of $1 \mathrm{~K}$. At $1 \mathrm{ps}$ we observe that the temperature range spanned by all the modes is already of about $45 \mathrm{~K}$. Since the electron temperature is still above $2000 \mathrm{~K}$ at $1 \mathrm{ps}$, phonons are further heated and their distribution broadens as shown at $5 \mathrm{ps}$. At this time the majority of the phonons are distributed nearly symmetrically around the averaged effective temperature within a range of $90 \mathrm{~K}$. At $10 \mathrm{ps}$, the averaged effective temperature of the phonons is nearly converged and the electron temperature is reduced to below $500 \mathrm{~K}$. The phonon distribution starts narrowing due to energy redistribution within the phonon system. The hottest phonons are cooling down by transferring their energy to the rest of the phonon system via phononphonon scatterings. At $15 \mathrm{ps}$, the temperature distribution becomes deltalike and the phonons can be considered nearly in thermal equilibrium. Nonetheless, a few modes are still below the averaged temperature, due to their low electronphonon and phonon-phonon coupling. This demonstrates that although phonon-phonon thermalization is very relevant in $\mathrm{Au}$, deviations from the two-temperature model still occur and a correct description of the lattice dynamics requires an out-of-equilibrium description. 

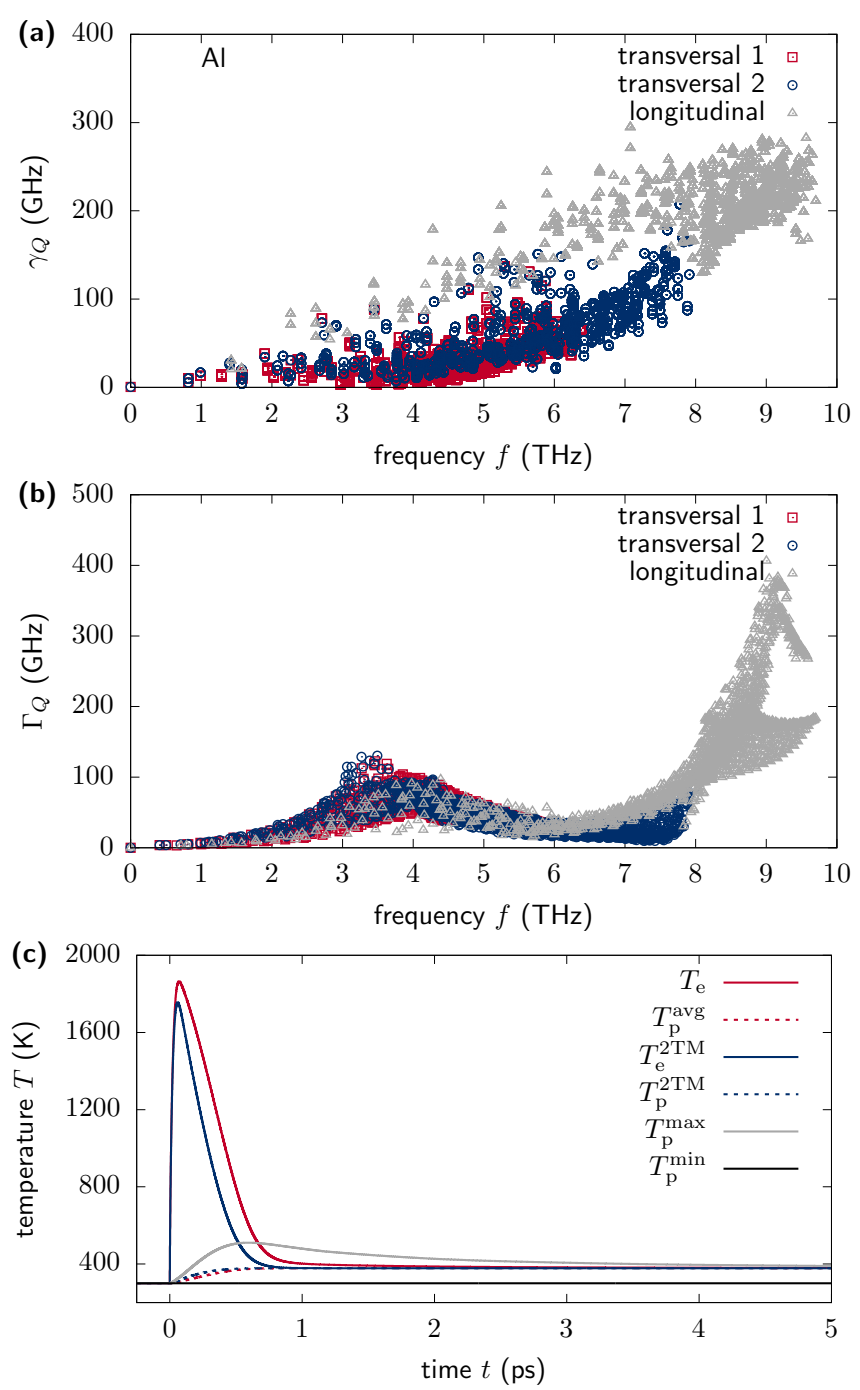

FIG. 4. (a) Calculated electron-phonon linewidths as a function of the phonon frequency for the three phonon branches in aluminum. (b) As (a), but for the phonon-phonon linewidths. (c) Comparison of the computed temperatures obtained from the two-temperature model with those of the microscopic out-of-equilibrium dynamics model as a function of time after laser excitation.

\section{B. Out-of-equilibrium dynamics in aluminum}

Unlike the case of gold, and as illustrated in Table I, aluminum has a larger electron-phonon coupling strength than the one provided by the phonon-phonon interaction, with an averaged value of about $101 \mathrm{GHz}$ and a lifetime of $4.9 \mathrm{ps}$. The phonon-phonon coupling is lower than in gold with an averaged linewidth of $77 \mathrm{GHz}$ corresponding to a lifetime of $6.5 \mathrm{ps}$. The mode-dependent linewidths for both cases are shown in Figs. 4(a) and 4(b). In contrast to the dependence found in other materials, the electron-phonon coupling varies strongly for the different phonon branches. Longitudinal phonon modes couple significantly stronger to electrons and also have on average a stronger phonon-phonon coupling than the other two branches. Similar results have been shown by Tang et al. [61].

The time evolution of electrons and averaged effective phonon temperatures in comparison with predictions from the

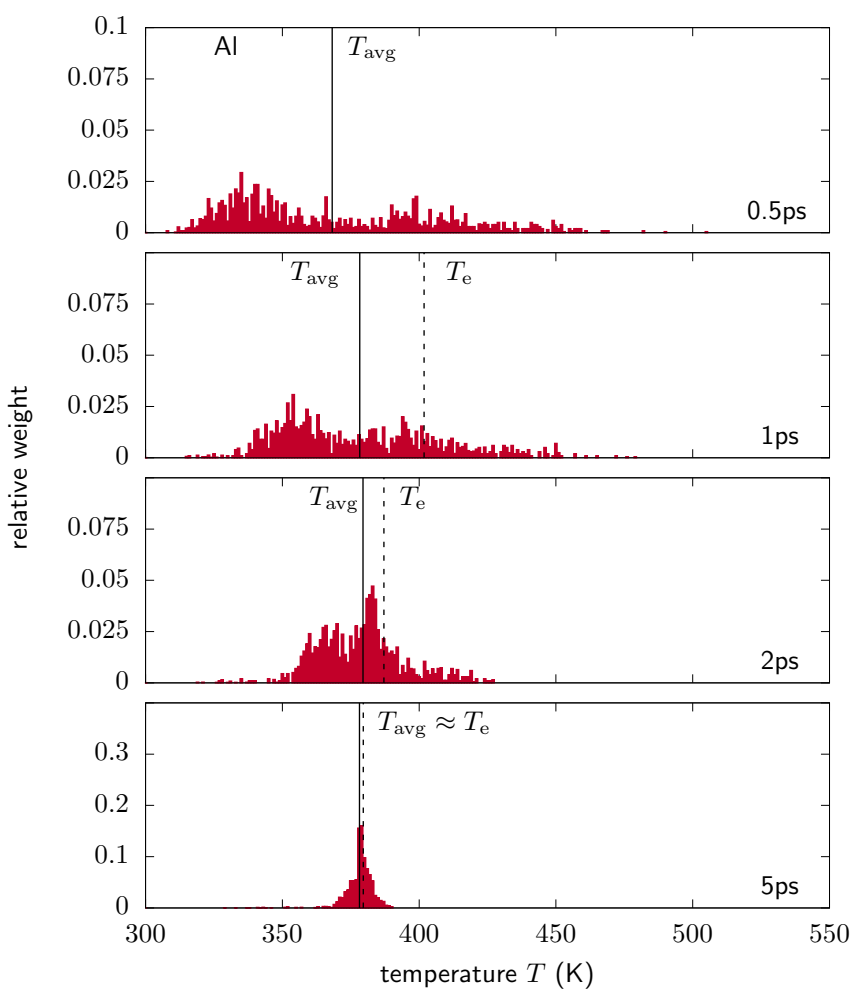

FIG. 5. Phononic temperature distribution at different times during electron-phonon relaxation in aluminum. The relative weights of phonon frequencies with a temperature within an interval of $1 \mathrm{~K}$ is shown for different times by the red bars.

two-temperature model are shown in Fig. 4(c). Electrons are heated up to above $1800 \mathrm{~K}$, whereas the relaxed temperature is again about $380 \mathrm{~K}$. The larger electron-phonon coupling, as compared with the gold quantities, leads to a faster relaxation than in the case of gold, having the averaged effective phonon temperature and electron temperature practically converged to a common value at $1 \mathrm{ps}$. However, it is important to emphasize that in this case we additionally observe larger deviations between the microscopic out-of-equilibrium dynamics model and the two-temperature model. In particular, the electron temperature is lower in the two-temperature model compared to the microscopic out-of-equilibrium dynamics model and the subsequent dynamic exhibits a faster relaxation.

We also calculate the temperature distributions during the different stages of the relaxation process and the results are shown in Fig. 5. The phonon distribution is much broader than for gold. Additionally, some phonon modes reach an effective temperature of about $100 \mathrm{~K}$ more than the averaged value. Below $2 \mathrm{ps}$, the distribution of the phonon temperatures is still very broad and asymmetric, evidencing a strong nonequilibrium state. The broad distribution results from the independent dynamics of the different phonon branches. The longitudinal phonon modes couple stronger to the electrons and among each other, whereas the other two branches have a lower electron-phonon coupling and a lower phonon-phonon coupling. This separates their dynamics and causes a very distinct dynamics for the different branches.

At $2 \mathrm{ps}$, the phonon distribution has narrowed. Since the electron temperature is in the range of the effective 
phonon temperatures, an indirect energy redistribution via electron-phonon coupling is possible. Although the electron temperature and the averaged effective phonon temperature converges at $1 \mathrm{ps}$, the phonons can only be considered to be in thermal equilibrium after around 5 ps. These results are in agreement with recent experimental observations and numerical results by Waldecker et al. [22]. In their work they assign the deviations from the two-temperature model to a phonon branch-dependent coupling to the electrons, with a phenomenological phonon-phonon coupling that is fitted to reproduce the experimental equilibration. However, it is relevant to stress that our results reveal an additional strong phonon frequency-dependent behavior, which leads to an even more complex dynamics and very broad effective phonontemperature distributions.

\section{Out-of-equilibrium dynamics in nickel}

The cases studied previously, for $\mathrm{Au}$ and $\mathrm{Al}$, show the capabilities of our model to describe the out-of-equilibrium lattice dynamics of nonmagnetic systems with very different electron-phonon and phonon-phonon couplings. In the following we will extend the description to study the relaxation dynamics of ferromagnetic materials, specifically nickel, iron, and cobalt. First, we compute the phonon linewidths due to electron-phonon coupling for the different materials by using spin-dependent densities of states and spin-dependent electron-phonon coupling. The mode-dependent linewidths in nickel are shown in Figs. 6(a) and 6(b). The averaged linewidth due to electron-phonon coupling is $272.5 \mathrm{GHz}$ equivalent to a lifetime of about $1.8 \mathrm{ps}$ which is significantly larger than for gold and aluminum (see Table I). The phononphonon linewidth in this material is on average only $27 \mathrm{GHz}$ leading to a lifetime of about $19 \mathrm{ps}$.

In Fig. 6(c) the temperature evolution of electrons and phonons computed for the two-temperature model and the microscopic out-of-equilibrium dynamics model are shown. Due to the strong electron-phonon coupling, the electrons are heated up within 100 fs to about $750 \mathrm{~K}$ and electrons and the averaged phonon temperature are nearly converged at about $0.75 \mathrm{ps}$ to a temperature of $349 \mathrm{~K}$. In Fig. 7 the effective phonon temperature distribution at different stages of the relaxation are shown. At $0.2 \mathrm{ps}$, a broad distribution with a range of $60 \mathrm{~K}$ is reached due to the mode-dependent coupling with the electrons. The maximum effective phonon temperature is much lower than in gold and aluminum and only about $380 \mathrm{~K}$. The maximum linewidth of electron-phonon coupling in aluminum is three times larger than the average value, whereas in nickel the maximum value is less than a factor 2 larger than the average. This leads to narrower phonon temperature distributions during the relaxation in nickel.

The thermalization of the phonons is mainly driven by electron-phonon coupling. Hotter phonons transfer their energy back to the electrons which are already colder than these phonons and this energy is again distributed to the less heated phonons. This is illustrated by the asymmetric temperature distributions at 1 and 2 ps in Fig. 7. The peak of the phonontemperature distribution is formed slightly above the electron temperature and it still deviates by several Kelvin from the averaged temperature. Hence, although the results provided
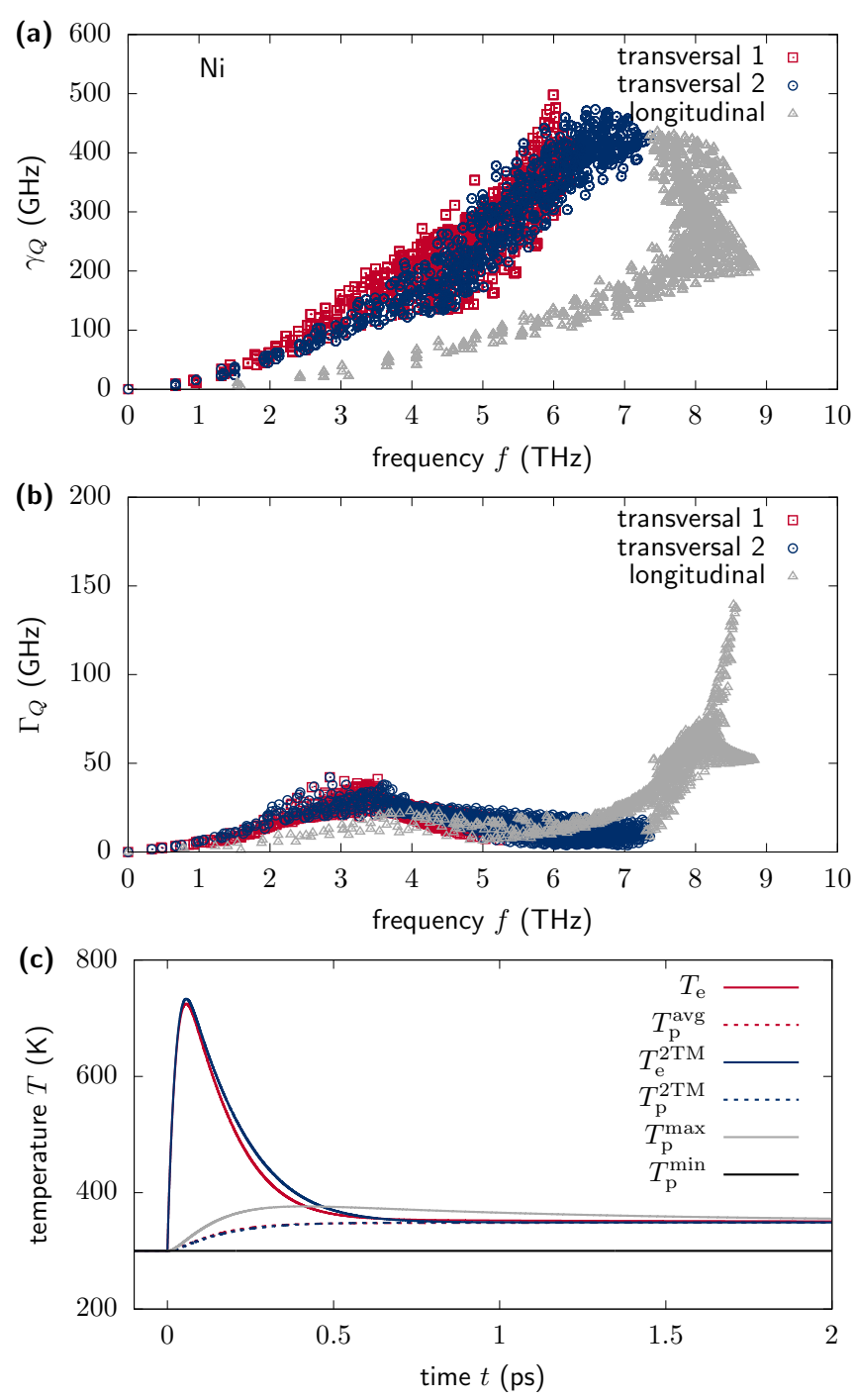

FIG. 6. (a) Calculated electron-phonon linewidths dependent on the phonon frequency for the three phonon branches in nickel. (b) As (a), but for the phonon-phonon linewidths. (c) Comparison of the computed temperatures obtained from the two-temperature model with those of the microscopic out-of-equilibrium dynamics model as a function of time after laser excitation.

by the two-temperature model and the calculated averaged dynamics of our model show a good agreement, the use of the two-temperature model leads to a wrong interpretation of the physical phenomena involved in the lattice thermalization in Ni.

\section{Out-of-equilibrium dynamics in iron}

Iron presents the lowest phonon linewidths due to phononphonon interaction among the here-discussed materials with an averaged value of $6.7 \mathrm{GHz}$ (equivalent to a lifetime of $75 \mathrm{ps}$ ) as shown in Table I. Additionally, the computed averaged electron-phonon coupling in iron is $164 \mathrm{GHz}$ (equivalent to $3 \mathrm{ps}$ lifetime), suggesting a larger relevance of this scattering mechanism in the dynamics than the phonon-phonon interaction. More specifically, this is illustrated in Figs. 8(a) and $8(\mathrm{~b})$, where the mode-dependent phonon linewidths are 


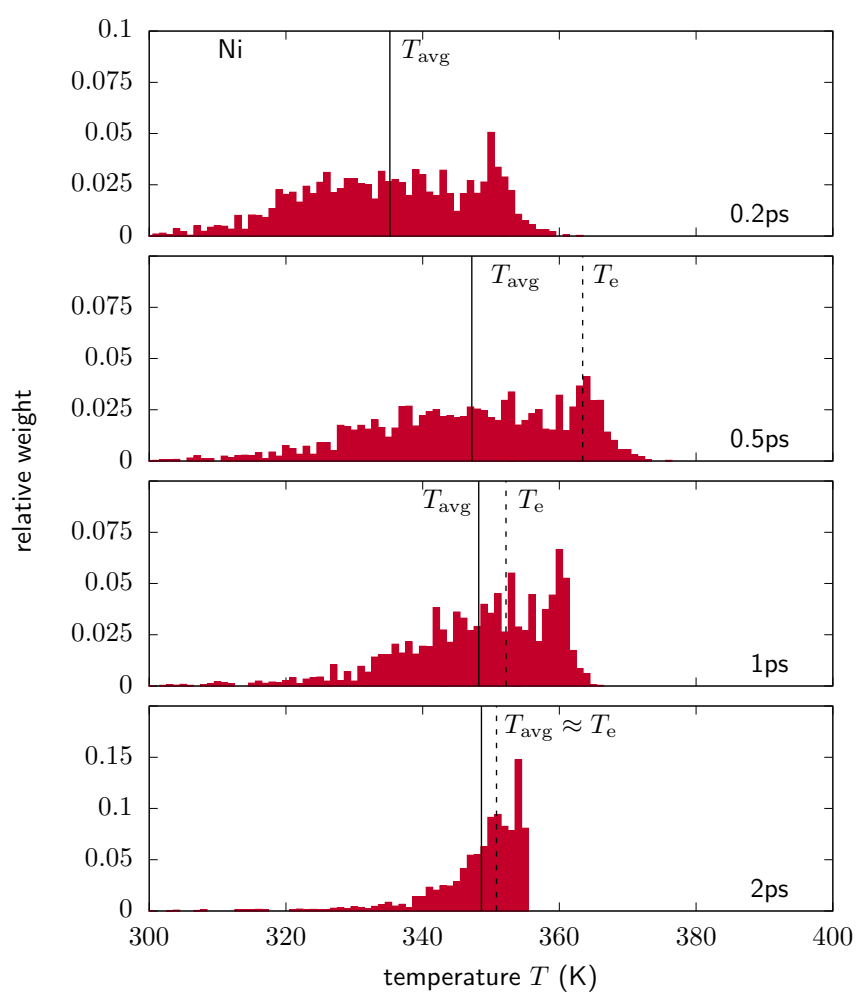

FIG. 7. Phononic temperature distribution at different times during electron-phonon relaxation in nickel. The red bars show the computed relative weights of phonon frequencies having a temperature within a range of $1 \mathrm{~K}$ for several different times. Vertical black lines give the average phonon temperature $T_{\text {avg }}$ and the vertical dashed line gives the electron temperature $T_{\mathrm{e}}$.

shown due to electron-phonon and phonon-phonon scatterings, respectively. The time evolution of the electron and phonon temperatures computed with the two-temperature model and the microscopic out-of-equilibrium dynamics model are summarized in Fig. 8(c). The results show that the two-temperature model provides a dynamics that is in significant contrast with the out-of-equilibrium model, showing a faster relaxation with an electronic temperature reaching a maximum value of around $200 \mathrm{~K}$ lower than in our model. Despite this discrepancy it is noteworthy to observe that the averaged effective phonon temperature in our model shows a very good agreement with the lattice temperature from the two-temperature model.

The distributions of the effective phonon temperatures during the relaxation are shown in Fig. 9. Unlike the case of nickel where the range of effective temperature deviations was about $70 \mathrm{~K}$, in iron the range of temperatures is above $100 \mathrm{~K}$ due to a strongly phonon mode-dependent electronphonon coupling. Moreover, the temperature distribution is very asymmetric with practically the same relative weight of phonons for all the temperatures spanned. As a consequence a large percentage of phonon modes have much larger temperatures than the averaged effective phonon temperature and, more importantly, larger than the electron temperature. This results in a flow of energy from those phonon modes to the electronic system which then deliver the energy to the phonon modes with lower temperatures. Since the phonon-phonon
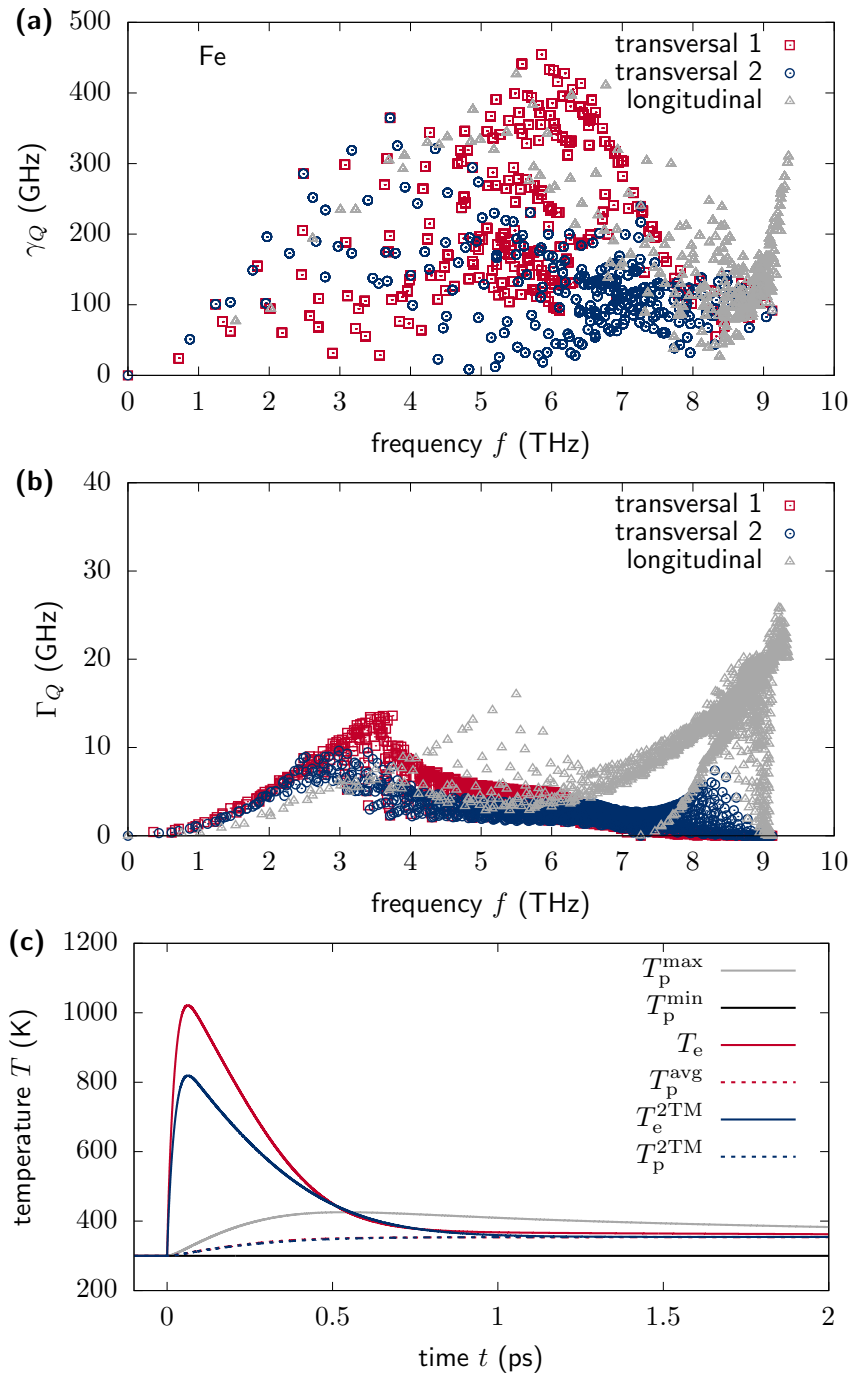

FIG. 8. (a) Calculated electron-phonon linewidths dependent on the phonon frequency for the three phonon branches in iron. (b) As (a), but for the phonon-phonon linewidths. (c) Comparison of the computed temperatures from the two-temperature model with those from the microscopic out-of-equilibrium dynamics model as a function of time after laser excitation.

coupling strength is small, it plays a small role in the lattice relaxation. Additionally, even though the averaged effective phonon temperature and the electron temperature are converged after $1 \mathrm{ps,} \mathrm{Fig.} 9$ shows that the phonon system remains in an out-of-equilibrium state for longer times and only after more than 5 ps can we start considering that the system is in a quasiequilibrium state.

\section{E. Out-of-equilibrium dynamics in cobalt}

As a last example we study the relaxation dynamics in hcp cobalt, which, as shown in Table I, has the largest electron-phonon coupling strength among all the systems here analyzed. Moreover, hcp cobalt has two inequivalent atoms in the primitive cell, which leads to six different phonon branches instead of three as in the other materials. The calculated phonon linewidths due to electron-phonon and 


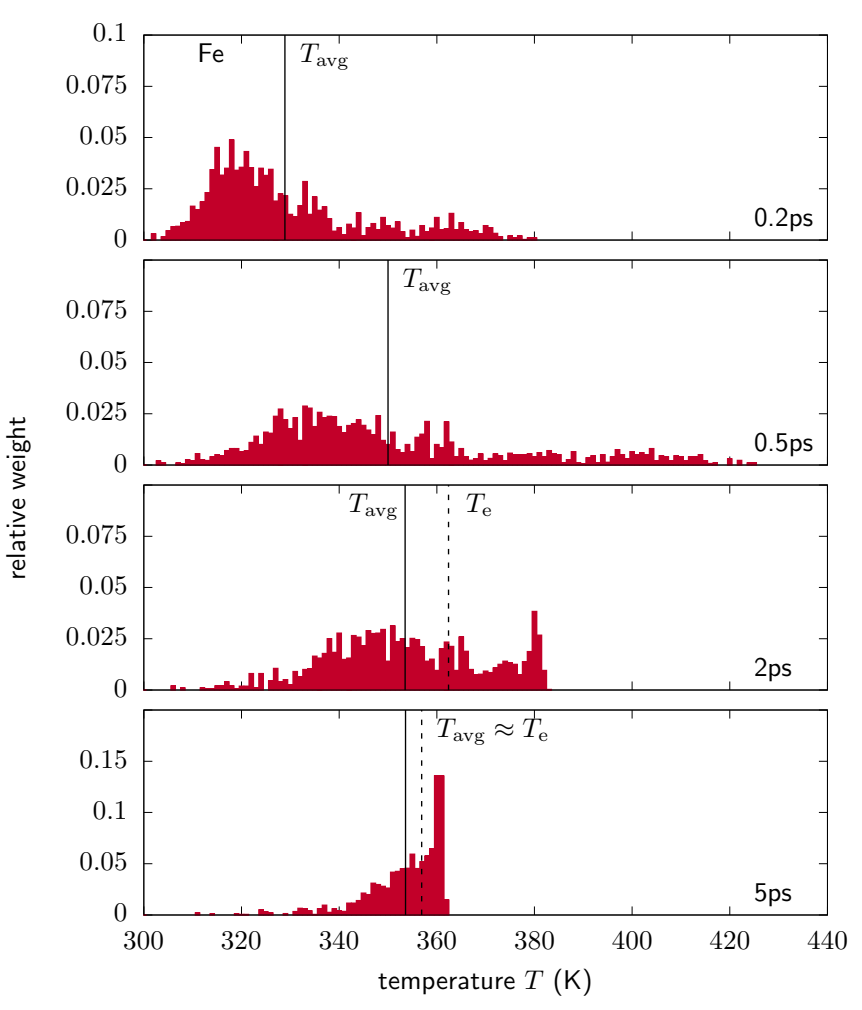

FIG. 9. Phononic temperature distribution at different times during electron-phonon relaxation in iron. The red bars show the computed relative weights of phonon frequencies having a temperature within an interval of $1 \mathrm{~K}$ is shown for several different times.

phonon-phonon couplings are shown in Figs. 10(a) and 10(b). The averaged electron-phonon linewidth is about $244.4 \mathrm{GHz}$ corresponding to a lifetime of only 2 ps and the averaged phonon-phonon linewidth is about $16.4 \mathrm{GHz}$ giving a lifetime of around $31 \mathrm{ps}$. The larger complexity of the primitive unit cell in Co, with respect to the other materials studied, favors a larger possibility for the electrons to interact with the phonons, leading to a very strong mode-dependent electronphonon coupling.

The temporal evolution of the effective temperatures after laser excitation is shown in Fig. 10(c). The twotemperature model deviates strongly from the microscopic out-of-equilibrium dynamics model. The maximum electron temperature in the microscopic out-of-equilibrium dynamics model remains below $900 \mathrm{~K}$, whereas the two-temperature model predicts a maximum electron temperature above $1200 \mathrm{~K}$. Also, the relaxation times of both models strongly deviate from each other. The electron and phonon temperatures of the two-temperature model converge already shortly after $0.1 \mathrm{ps,}$, whereas the averaged effective phonon temperature and the electron temperature in the microscopic out-of-equilibrium dynamics model converge at about $0.2 \mathrm{ps}$. The final temperature is much lower than in the other cases caused by the higher heat capacity of the phonons due to the larger number of phonon branches.

In contrast to the other two ferromagnetic metal systems, the effective phononic temperature distribution in cobalt is narrower as shown in Fig. 11. The overall heating effect in the phononic system is smaller than for the other cases studied
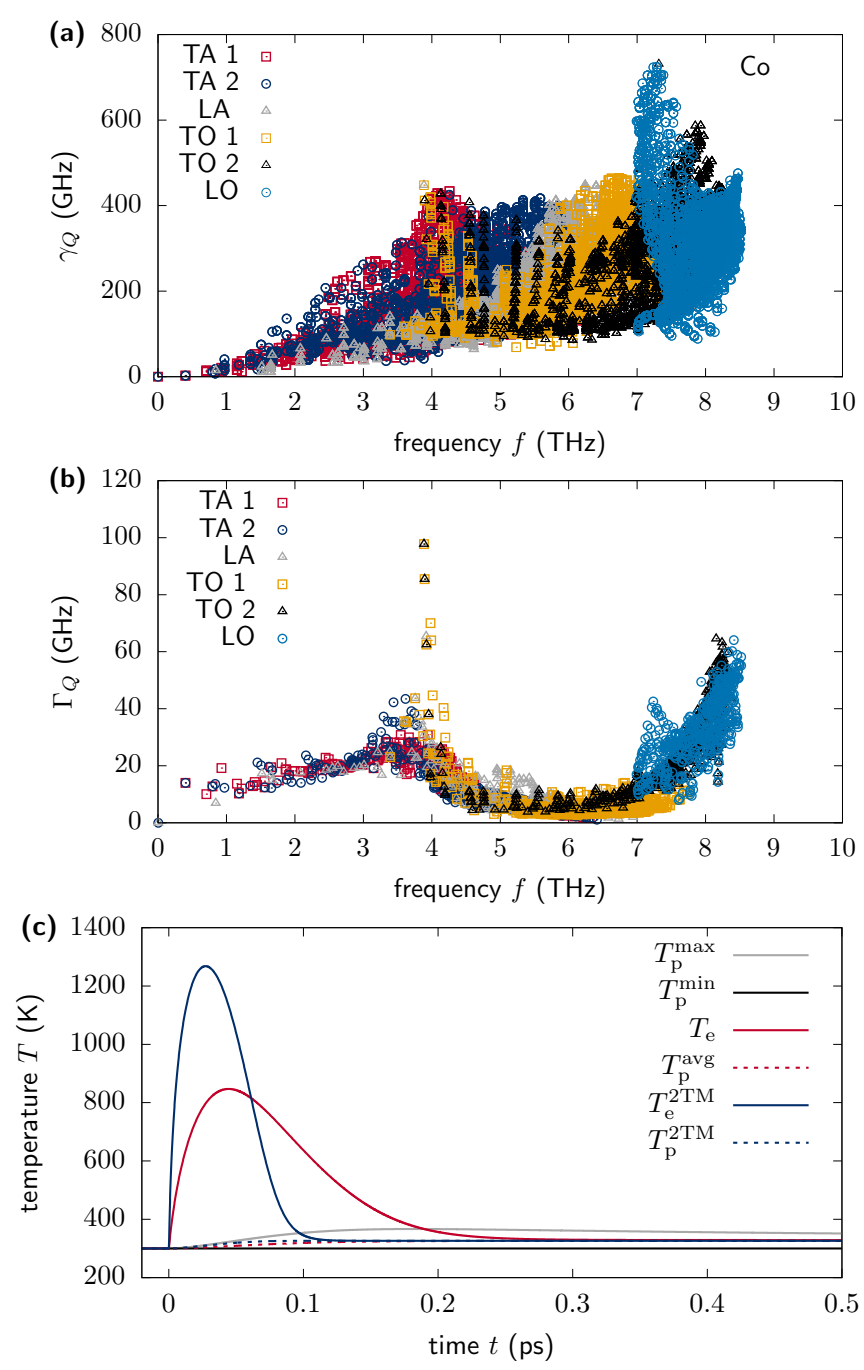

FIG. 10. (a) Calculated electron-phonon linewidths dependent on the phonon frequency for the three phonon branches in cobalt. (b) As (a), but for the phonon-phonon linewidths. (c) Comparison of the computed temperatures obtained from the two-temperature model with those of the microscopic out-of-equilibrium dynamics model as a function of time after laser excitation.

here due to the large phonon heat capacity. The broadest distribution is already obtained before $0.2 \mathrm{ps}$ and the effective phonon temperature range is about almost $80 \mathrm{~K}$. It is important to mention that during the timescale at which the system's evolution occurs the electrons remain out-of-equilibrium, and therefore the assumption of thermal electrons is not well justified and probably requires a more advanced description that is beyond the scope of this work.

\section{DISCUSSION AND CONCLUSIONS}

We have applied a microscopic out-of-equilibrium model to study the laser-induced relaxation dynamics in five different metals, i.e., gold, aluminum, nickel, iron, and cobalt. As a general observation we have evidenced that the two-temperature model fails to describe accurately the lattice dynamics and, in some cases as in cobalt and iron, also the electronic dynamics. Surprisingly, this is evidenced even in the case of gold, 


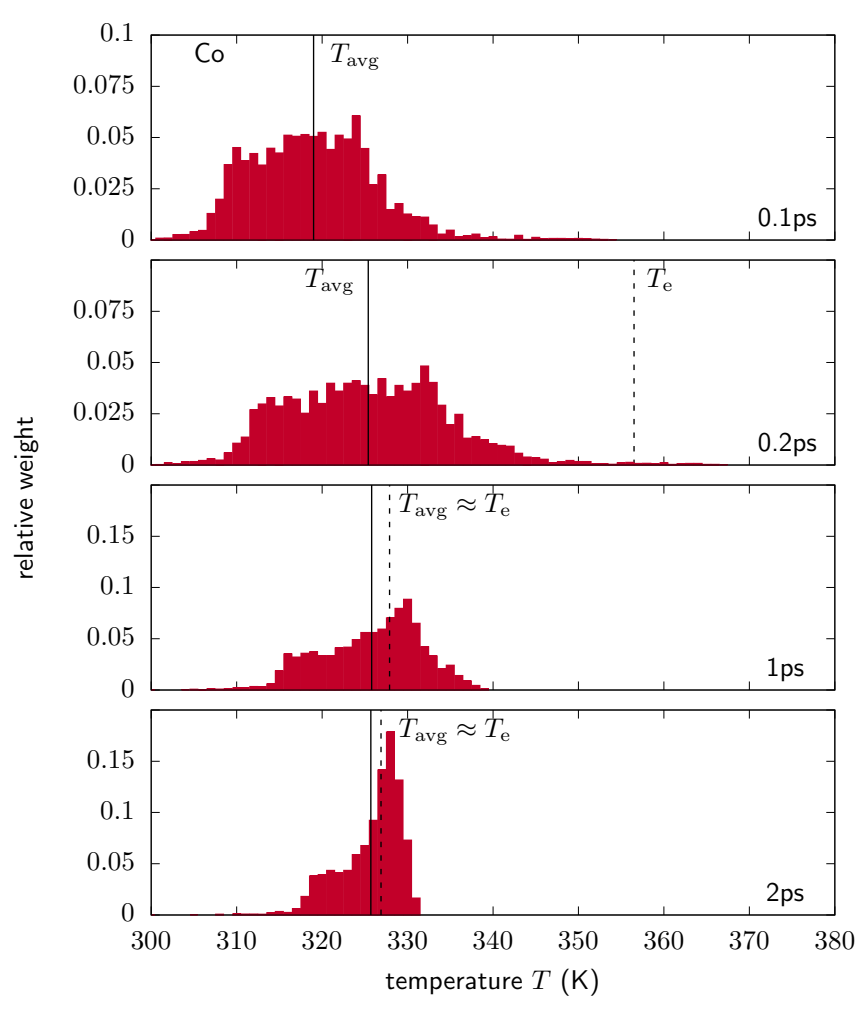

FIG. 11. Phononic temperature distribution at different times during electron-phonon relaxation in hcp cobalt. The red bars show the computed relative weights of phonon frequencies having a temperature within an interval of $1 \mathrm{~K}$ for several different times.

where the assumptions on which the two-temperature model are based would seem better justified.

To describe the out-of-equilibrium state more quantitatively we calculate the mean deviation from the timedependent averaged effective phonon temperature $\Delta_{\text {avg }}=$ $\sum_{Q}\left(\left|T_{Q}-T_{\text {avg }}\right|\right) / N_{Q}$. A comparison of the resulting deviation as a function of time is shown in Fig. 12. In all cases the mean deviation first increases when the electron temperature is higher than the effective phonon temperatures and then decays on different timescales. Due to the strong phononphonon coupling in gold, the mean deviation does not exceed $12 \mathrm{~K}$, but due to the small electron-phonon coupling, some deviations still appear on a timescale longer than 5 ps. The

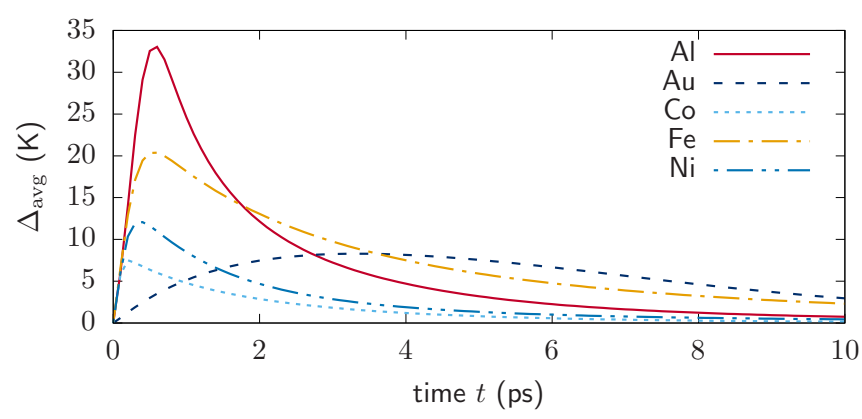

FIG. 12. Computed mean deviation from the time-dependent averaged phonon temperature as a function of time for all materials studied.

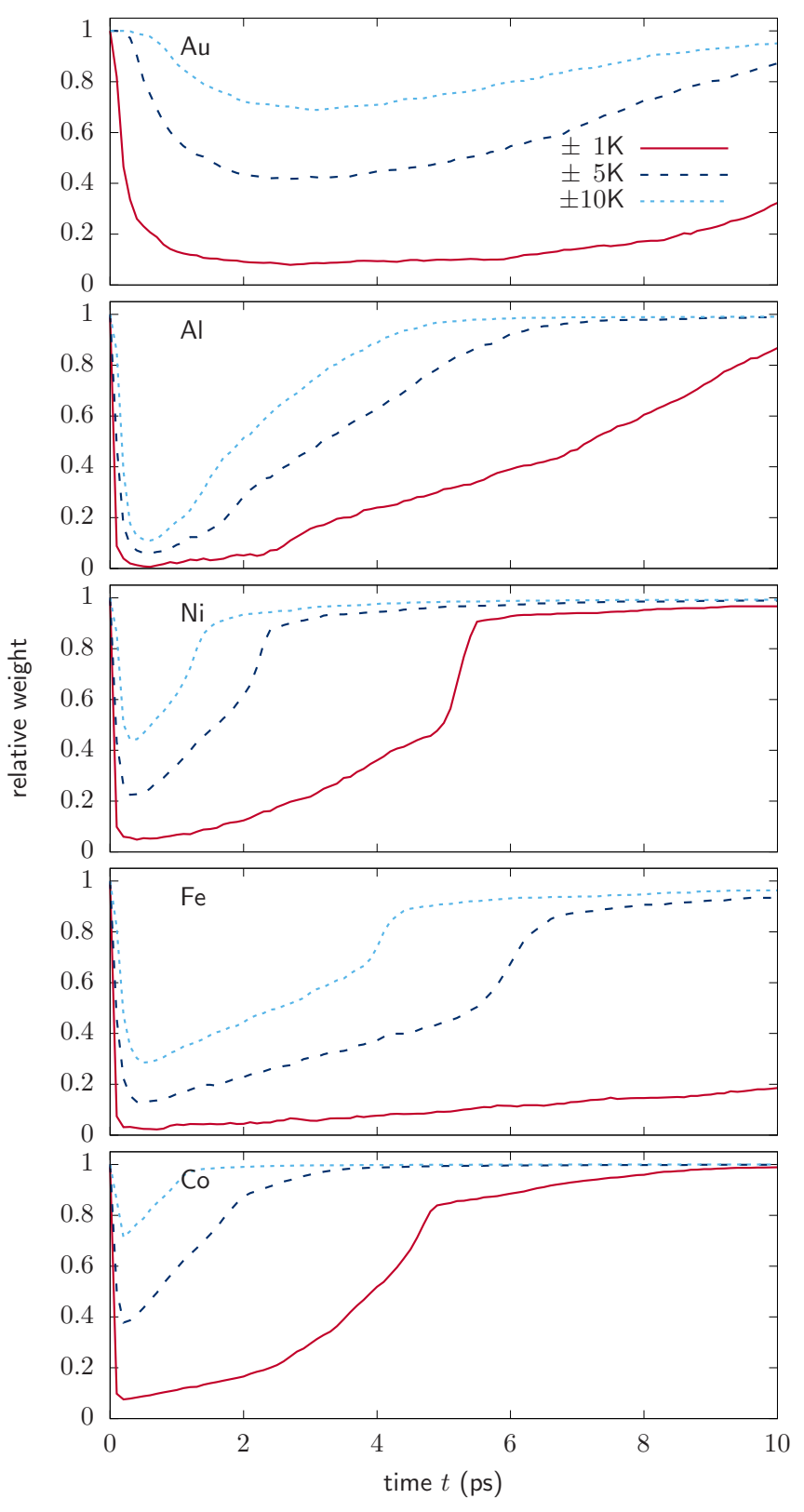

FIG. 13. Relative weight of phonon frequencies within a range of $\pm 1 \mathrm{~K}$ (red curve), $\pm 5 \mathrm{~K}$ (dark blue curve), and $\pm 10 \mathrm{~K}$ (light blue curve) around the averaged phonon temperature as a function of time for the different metals considered.

largest mean deviation is obtained in aluminum for which the mean deviation is around $33 \mathrm{~K}$ after $1 \mathrm{ps}$ of the laser excitation, evidencing a very spread distribution of phonon temperatures. In the ferromagnetic metals iron, nickel, and cobalt the mean deviation is always below $20 \mathrm{~K}$ for the laser intensities used. In these cases, the averaged phonon linewidth due to electron-phonon coupling is larger than in aluminum, but the relative deviations from the averaged linewidth are smaller. Therefore, in aluminum we obtain larger values for the mean deviations indicating a state far from equilibrium, whereas in other materials the mean deviations are lower and the phononic systems are not driven as far from equilibrium. Nonetheless, these data underline that the assumption of 
thermalized phonon distributions is not fulfilled on the picosecond timescale for all materials. As a last step we evaluate how good the description of the averaged phonon temperature captures the overall behavior of the lattice relaxation. For that purpose we consider the relative amount of phonon modes around the averaged temperature within a range $\pm 1, \pm 5$, and $\pm 10 \mathrm{~K}$, which is shown for all materials in Fig. 13. The relative number of effective phonon temperatures around the averaged effective temperature first drops strongly and then smoothly recovers. Aside from the different timescales of the relaxation, the dynamical behavior itself differs between the materials. The relative number of phonons within a range of $10 \mathrm{~K}$ around the averaged temperature in gold drops to about $80 \%$ which means that the averaged effective temperature represents the overall behavior of the majority of the modes. But in aluminum the value drops to only $15 \%$ and the averaged temperature cannot be considered at all as a good description for the system. Afterwards the value increases to almost 1 at around $5 \mathrm{ps}$, whereas the number increases slower in gold and is still below 1 at $10 \mathrm{ps.}$

In the ferromagnetic metals we observe a different behavior. For iron and nickel the number of phonon modes around the averaged effective temperature with a difference up to $10 \mathrm{~K}$ decreases below $50 \%$ and recovers on different material specific timescales. One can see that at certain times jumps occur, where the relative number around the averaged effective temperature strongly increases. This indicates that larger fractions of phonon modes have acquired similar effective temperatures that deviate from the averaged value. During thermalization the effective temperature of those modes converges towards the averaged temperature leading to a large increase of the relative amount of modes with an effective temperature around the averaged value. In cobalt, the drop of the relative weight is less strong for the \pm 5 and $\pm 10 \mathrm{~K}$ intervals, again due to the large heat capacity of the phonons.

In summary, we have shown strong out-of-equilibrium phononic distributions after laser excitation on the picosecond timescale demonstrating the limitations of the two-temperature model on that timescale. Our microscopic out-of-equilibrium dynamics model based on an $a b$ initio input parameter such as phonon mode dependent electron-phonon coupling and phonon-phonon coupling demonstrate that the obtained out-of-equilibrium dynamics are very material specific and the phonon-mode dependencies of the coupling processes have to be considered. In aluminum we have found the strongest deviations from equilibrium due to the decoupling of the different phonon branches. It was proposed earlier by Waldecker et al. to use a different coupling for each branch [22], which can improve the description of the dynamics. Our findings show that this works only in certain materials such as aluminum and although this can improve the description, it lacks still the strong mode-dependent electron-phonon coupling within each branch.

Our simulations clearly show that the temperature of the hottest phonons can exceed the electron temperature and relax on longer timescales than the electrons. This is in contrast to a simplified model for electron-phonon relaxation by Sadasivam et al. [38], in which they assume that the hottest phonons would follow the electron temperature. Such an assumption would exclude a possible energy redistribution of the highly excited phonon modes to lower excited ones via electron-phonon coupling. Through our explicit calculations, we demonstrate that this relaxation channel plays an important role in the case of strong electron-phonon coupling and weak phonon-phonon interaction.

All this previously unnoted behavior exemplifies that the two-temperature model is an insufficient description of the relaxation process of electrons and phonons after laser excitation in the picosecond time range and mode-dependent dynamics have to be considered in order to describe the heating process and subsequent system dynamics on that timescale.

\section{ACKNOWLEDGMENTS}

We thank R. Ernstorfer for valuable discussions. The authors acknowledge financial support from the Deutsche Forschungsgemeinschaft via RI 2891/1-1, RI 2891/2-1, and TRR 227 "Ultrafast Spin Dynamics," as well as from the Swedish Research Council (VR), and the Knut and Alice Wallenberg Foundation (Grant No. 2015.0060). We also acknowledge support from the Swedish National Infrastructure for Computing (SNIC).

\section{APPENDIX A: MICROSCOPIC PHONON-PHONON SCATTERING TERM}

The scattering term for the phonon population due to phonon-phonon interaction can be written as [46]

$$
\begin{aligned}
\left.\dot{n}_{Q}\right|_{\mathrm{ph}-\mathrm{ph}} ^{\text {scatt. }}=\frac{2 \pi}{\hbar^{2}} \frac{36}{2} \sum_{k, k^{\prime}}\left|\Phi_{-Q, k, k^{\prime}}\right|^{2}\left[\left(n_{Q}+1\right)\left(n_{k}+1\right) n_{k^{\prime}} \delta\left(\omega_{Q}+\omega_{k^{\prime}}-\omega_{k^{\prime \prime}}\right)+\left(n_{Q}+1\right)\left(n_{k^{\prime}}+1\right) n_{k} \delta\left(\omega_{Q}+\omega_{k^{\prime}}-\omega_{k}\right)\right. \\
-n_{Q} n_{k}\left(n_{k^{\prime}}+1\right) \delta\left(\omega_{Q}-\omega_{k^{\prime}}+\omega_{k}\right)-n_{Q} n_{k^{\prime}}\left(n_{k}+1\right) \delta\left(\omega_{Q}+\omega_{k^{\prime}}-\omega_{k}\right) \\
\left.+\left(n_{Q}+1\right) n_{k} n_{k^{\prime}} \delta\left(\omega_{Q}-\omega_{k^{\prime}}-\omega_{k}\right)-n_{Q}\left(n_{k}+1\right)\left(n_{k^{\prime}}+1\right) \delta\left(\omega_{Q}-\omega_{k^{\prime}}-\omega_{k}\right)\right] .
\end{aligned}
$$

The factor $\frac{36}{2}$ is introduced due to the (3!) $)^{2}$ equivalent terms from the summation in the phonon-phonon matrix elements and the factor $1 / 2$ to avoid double counting in the summation in Eq. (A1). We can now use the following relations [and additionally, we define $\beta(k) \equiv \frac{\hbar \omega_{k}}{k_{\mathrm{B}} T_{k}}$ ]:

$$
\begin{gathered}
n_{k} n_{k^{\prime}}=\frac{1}{\left(e^{\beta(k)}-1\right)\left(e^{\beta\left(k^{\prime}\right)}-1\right)}=\left(n_{k}+n_{k^{\prime}}+1\right) \frac{1}{e^{\beta(k)+\beta\left(k^{\prime}\right)}-1}, \\
n_{k}\left(n_{k^{\prime}}+1\right)=\frac{1}{e^{\beta(k)}-1}\left(\frac{1}{e^{\beta\left(k^{\prime}\right)}-1}+1\right)=\left(n_{k}-n_{k^{\prime}}\right) \frac{-1}{e^{\beta(k)-\beta\left(k^{\prime}\right)}-1} .
\end{gathered}
$$


and then Eqs. (A2) and (A3) become

$$
\begin{gathered}
n_{k} n_{k^{\prime}}=\left(n_{k}+n_{k^{\prime}}+1\right) n_{Q}\left(\beta_{0}\right), \quad \text { for } \delta\left(\omega_{Q}-\omega_{k^{\prime}}-\omega_{k}\right), \\
n_{k}\left(n_{k^{\prime}}+1\right)=-\left(n_{k}-n_{k^{\prime}}\right) n_{Q}\left(\beta_{1}\right), \quad \text { for } \delta\left(\omega_{Q}+\omega_{k^{\prime}}-\omega_{k}\right), \\
n_{k^{\prime}}\left(n_{k}+1\right)=-\left(n_{k^{\prime}}-n_{k}\right) n_{Q}\left(\beta_{2}\right), \quad \text { for } \delta\left(\omega_{Q}-\omega_{k^{\prime}}+\omega_{k}\right),
\end{gathered}
$$

with

$$
\begin{aligned}
& \beta_{0}\left(k, k^{\prime}, Q\right)=\beta_{0}=\frac{\hbar \omega_{k}\left(T_{k^{\prime}}-T_{k}\right)+\hbar \omega_{Q} T_{k}}{k_{\mathrm{B}} T_{k} T_{k^{\prime}}}=\frac{\hbar \omega_{Q}}{k_{\mathrm{B}} T_{k^{\prime}}}\left(1+\frac{\omega_{k}}{\omega_{Q}} \frac{T_{k^{\prime}}-T_{k}}{T_{k}}\right)=\frac{\hbar \omega_{Q}}{k_{\mathrm{B}} T_{k}}\left(1+\frac{\omega_{k^{\prime}}}{\omega_{Q}} \frac{T_{k}-T_{k^{\prime}}}{T_{k^{\prime}}}\right), \\
& \beta_{1}\left(k, k^{\prime}, Q\right)=\beta_{1}=\frac{\hbar \omega_{k}\left(T_{k^{\prime}}-T_{k}\right)+\hbar \omega_{Q} T_{k}}{k_{\mathrm{B}} T_{k} T_{k^{\prime}}}=\frac{\hbar \omega_{Q}}{k_{\mathrm{B}} T_{k^{\prime}}}\left(1+\frac{\omega_{k}}{\omega_{Q}} \frac{T_{k^{\prime}}-T_{k}}{T_{k}}\right)=\frac{\hbar \omega_{Q}}{k_{\mathrm{B}} T_{k}}\left(1+\frac{\omega_{k^{\prime}}}{\omega_{Q}} \frac{T_{k^{\prime}}-T_{k}}{T_{k^{\prime}}}\right), \\
& \beta_{2}\left(k, k^{\prime}, Q\right)=\beta_{2}=\frac{\hbar \omega_{k}\left(T_{k}-T_{k^{\prime}}\right)+\hbar \omega_{Q} T_{k}}{k_{\mathrm{B}} T_{k} T_{k^{\prime}}}=\frac{\hbar \omega_{Q}}{k_{\mathrm{B}} T_{k^{\prime}}}\left(1-\frac{\omega_{k}}{\omega_{Q}} \frac{T_{k^{\prime}}-T_{k}}{T_{k}}\right)=\frac{\hbar \omega_{Q}}{k_{\mathrm{B}} T_{k}}\left(1-\frac{\omega_{k^{\prime}}}{\omega_{Q}} \frac{T_{k^{\prime}}-T_{k}}{T_{k^{\prime}}}\right) .
\end{aligned}
$$

Finally, Eq. (A1) can be written as

$$
\begin{gathered}
\left.\dot{n}_{Q}\right|_{\mathrm{ph}-\mathrm{ph}} ^{\text {scatt. }}=\frac{36 \pi}{\hbar^{2}} \sum_{k, k^{\prime}}\left|\Phi_{-Q, k, k^{\prime}}\right|^{2}\left[\left(n_{k}-n_{k^{\prime}}\right)\left\{\delta\left(\omega_{Q}+\omega_{k}-\omega_{k^{\prime}}\right)\left[n\left(\beta_{2}\right)-n_{Q}\right]-\delta\left(\omega_{Q}-\omega_{k}+\omega_{k^{\prime}}\right)\left[n\left(\beta_{1}\right)-n_{Q}\right]\right\}\right. \\
\left.+\left[n\left(\beta_{0}\right)-n_{Q}\right]\left(n_{k}+n_{k^{\prime}}+1\right) \delta\left(\omega_{Q}-\omega_{k}-\omega_{k^{\prime}}\right)\right] \\
=\frac{36 \pi}{\hbar^{2}} \sum_{k, k^{\prime}}\left|\Phi_{-Q, k, k^{\prime}}\right|^{2}\left[\left(n_{k}-n_{k^{\prime}}\right)\left[n\left(\beta_{2}\right) \delta\left(\omega_{Q}+\omega_{k}-\omega_{k^{\prime}}\right)-n\left(\beta_{1}\right) \delta\left(\omega_{Q}-\omega_{k}+\omega_{k^{\prime}}\right)\right]\right. \\
\left.+n\left(\beta_{0}\right)\left(n_{k}+n_{k^{\prime}}+1\right) \delta\left(\omega_{Q}-\omega_{k}-\omega_{k^{\prime}}\right)\right] .
\end{gathered}
$$

Then, depending on the cases considered, $\tilde{T}_{\mathrm{p}}{ }^{k, k^{\prime}}$ defined in Eqs. (11) and (12) will be equal to $\tilde{T}_{\mathrm{p}}^{k, k^{\prime}}=T_{k}\left(1-\frac{\omega_{k^{\prime}}}{\omega_{Q}} \frac{T_{k^{\prime}}-T_{k}}{T_{k^{\prime}}}\right)^{-1}$ or $\tilde{T}_{\mathrm{p}}^{k, k^{\prime}}=T_{k}\left(1+\frac{\omega_{k^{\prime}}}{\omega_{Q}} \frac{T_{k^{\prime}}-T_{k}}{T_{k^{\prime}}}\right)^{-1}$.

From Eq. (A11) we can reach Eq. (12) as derived in Maldonado et al. [36] by Taylor expanding the phonon distributions about the phonon temperature difference.

On the other hand, the phonon linewidth $\gamma_{Q}$ due to electron-phonon coupling and phonon linewidth $\Gamma_{Q k^{\prime}}$ due to phonon-phonon scattering are given by (see also Ref. [36])

$$
\begin{aligned}
\gamma_{Q}=2 \pi & \omega_{Q} \sum_{k \sigma}\left|g_{n n^{\prime} \mathbf{k}^{\prime}}^{\sigma}(\mathbf{k}, \mathbf{q})\right|^{2} \delta\left(\epsilon_{\mathbf{k}^{\prime} n^{\prime}}^{\sigma}-\epsilon_{\mathrm{F}}\right) \delta\left(\epsilon_{\mathbf{k} n}^{\sigma}-\epsilon_{\mathrm{F}}\right), \\
\Gamma_{Q, k}= & \frac{18 \pi}{\hbar^{2}}\left|\Phi_{-Q, k, k^{\prime}}\right|^{2}\left\{\left(n_{k}-n_{k^{\prime}}\right)\right. \\
& \times\left[\delta\left(\omega_{Q}+\omega_{k}-\omega_{k^{\prime}}\right)-\delta\left(\omega_{Q}-\omega_{k}+\omega_{k^{\prime}}\right)\right] \\
& \left.+\left(n_{k}+n_{k^{\prime}}+1\right) \delta\left(\omega_{Q}-\omega_{k}-\omega_{k^{\prime}}\right)\right\} .
\end{aligned}
$$

Here $g_{n n^{\prime} \mathbf{k}^{\prime}}^{\sigma}(\mathbf{k}, \mathbf{q})$ and $\Phi_{-Q, k, k^{\prime}}$ denote electron-phonon and phonon-phonon matrix elements and $\epsilon_{\mathbf{k}^{\prime} n^{\prime} \sigma}$ is the energy of the electron with momentum $\mathbf{k}^{\prime}$, electron band $n^{\prime}$ and spin $\sigma$, and $\epsilon_{\mathrm{F}}$ is the Fermi energy. Although the transient changes of the phonon population depend explicitly on the changing mode-dependent phonon distributions, the linewidths in Eqs. (A12) and (A13) can still be computed using phonon modes at equilibrium for the cases of low laser fluences, where this approximation provides results equivalent to using time-dependent out-of-equilibrium phonon linewidths.

The quantity $I\left(T_{\mathrm{e}}\right)$ introduced in Eqs. (11) and (12) is a correction factor for the electron-phonon coupling accounting for scattering of electrons away from the Fermi surface. It can be computed by

$$
I\left(T_{\mathrm{e}}\right)=-\int_{-\infty}^{\infty} d \epsilon \frac{\partial f_{k}}{\partial \epsilon} \frac{g(\epsilon)^{2}}{g\left(\epsilon_{\mathrm{F}}\right)^{2}},
$$

where $g(\epsilon)$ is the electron density of states at energy $\epsilon$. The quantity $J\left(\omega_{Q}, T_{\mathrm{p}}^{Q}\right)$ in Eqs. (11) and (12) represents the second-order term in a Taylor expansion in temperature differences of the out-of-equilibrium phonon population

$$
J\left(\omega_{Q}, T_{\mathrm{p}}^{Q}\right)=\frac{\hbar \omega_{Q}}{k_{\mathrm{B}} T_{\mathrm{p}}^{2}}\left(\frac{\exp \left(\frac{\hbar \omega_{Q}}{k_{\mathrm{B}} T_{\mathrm{p}}^{Q}}\right)+1}{\exp \left(\frac{\hbar \omega_{Q}}{k_{\mathrm{B}} T_{\mathrm{p}}^{Q}}\right)-1}-\frac{2 k_{\mathrm{B}} T_{\mathrm{p}}^{Q}}{\hbar \omega_{Q}}\right) .
$$

\section{APPENDIX B: FIRST-PRINCIPLES CALCULATIONS}

The electron-phonon coupling constants were computed as response function quantities using the density-functional perturbation theory (DFPT) as implemented in ABINIT [59].

The harmonic phonon dispersion and the anharmonic phonon properties were obtained by performing calculations with the finite displacement method using the PHONO3PY package [47] and the Vienna $a b$ initio simulation package (VASP) [62] employed as the ab initio code to calculate the pairwise and cubic interatomic force constants. 
The phonon linewidths due to phonon-phonon interaction were determined using many-body perturbation theory on a third-order anharmonic Hamiltonian which included up to three-phonon scatterings [47]. Under these conditions the phonon linewidth computation reduces to knowing the thirdorder anharmonic interatomic force constants which can be determined from density functional theory calculations. To evaluate the anharmonic interatomic force constants we employ supercells consisting of $4 \times 4 \times 4$ primitive cells.

\section{APPENDIX C: MODE-DEPENDENT PHONON DISTRIBUTION IN GOLD}

As an addition to the effective phonon temperature distribution at different time delays, given in Sec. III A, we discuss here which phonon modes show high or low effective temperatures for the exemplary case of gold. Similar observations can be made for the other materials. In Fig. 14 we show the computed effective phonon temperature as a function of the frequency of the phonon modes for two different times delays. In the top panel the results are shown at $5 \mathrm{ps}$ after laser pumping. The effective temperature distribution is nonhomogeneous which evidences that the phonon system is clearly out-of-equilibrium. It is important to note that the obtained effective temperature of each mode results directly from the mode-dependent electronphonon coupling strength. Modes that couple efficiently to the electrons obtain the highest effective temperatures and are typically modes with higher frequencies. The lowest effective temperatures occur for phonon modes with lower energies, which, correspondingly, also exhibit a weak electron-phonon coupling.

In the bottom panel of Fig. 14 we show the effective temperatures at a $10 \mathrm{ps}$ delay after laser excitation. The results display a clear convergence to an almost final, thermalized phonon temperature, evidencing that the system

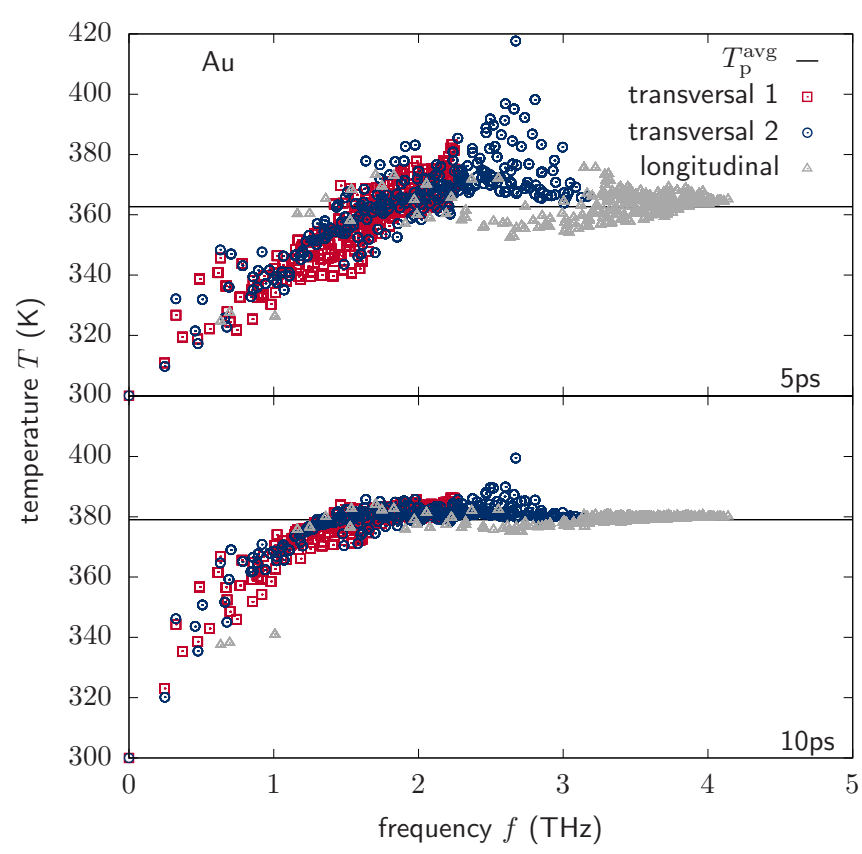

FIG. 14. Effective phonon temperatures computed as function of the frequency for gold at 5 ps (top panel) and 10 ps (bottom panel) after laser excitation.

is near thermalization. The phonon thermalization in gold is mainly driven by phonon-phonon interaction. Thus, the phonon modes that present large phonon-phonon coupling strength - mainly high frequency phonons-have reached effective phonon temperatures close to the average effective temperature. On the other hand, phonon modes having weak phonon-phonon coupling strength and low frequencies correspondingly have effective temperatures that deviate from, and are typically below, the average effective temperature.
[1] C.-K. Sun, F. Vallée, L. Acioli, E. P. Ippen, and J. G. Fujimoto, Phys. Rev. B 48, 12365 (1993).

[2] R. H. M. Groeneveld, R. Sprik, and A. Lagendijk, Phys. Rev. B 51, 11433 (1995).

[3] L. Stojchevska, I. Vaskivskyi, T. Mertelj, P. Kusar, D. Svetin, S. Brazovskii, and D. Mihailovic, Science 344, 177 (2014).

[4] J. D. Rameau, S. Freutel, A. F. Kemper, M. A. Sentef, J. K. Freericks, I. Avigo, M. Ligges, L. Rettig, Y. Yoshida, H. Eisaki, J. Schneeloch, R. D. Zhong, Z. J. Xu, G. D. Gu, P. D. Johnson, and U. Bovensiepen, Nat. Commun. 7, 13761 (2016).

[5] E. Beaurepaire, J.-C. Merle, A. Daunois, and J.-Y. Bigot, Phys. Rev. Lett. 76, 4250 (1996).

[6] J. Y. Bigot, M. Vomir, and E. Beaurepaire, Nat. Phys. 5, 515 (2009).

[7] A. Kirilyuk, A. V. Kimel, and T. Rasing, Rev. Mod. Phys. 82, 2731 (2010).

[8] F. Hansteen, A. Kimel, A. Kirilyuk, and T. Rasing, Phys. Rev. Lett. 95, 047402 (2005).

[9] J.-W. Kim, M. Vomir, and J.-Y. Bigot, Phys. Rev. Lett. 109, 166601 (2012).
[10] T. Henighan, M. Trigo, S. Bonetti, P. Granitzka, D. Higley, Z. Chen, M. P. Jiang, R. Kukreja, A. Gray, A. H. Reid, E. Jal, M. C. Hoffmann, M. Kozina, S. Song, M. Chollet, D. Zhu, P. F. Xu, J. Jeong, K. Carva et al., Phys. Rev. B 93, 220301(R) (2016).

[11] M. Harmand, R. Coffee, M. R. Bionta, M. Chollet, D. French, D. Zhu, D. M. Fritz, H. T. Lemke, N. Medvedev, B. Ziaja, S. Toleikis, and M. Cammarata, Nat. Photon. 7, 215 (2013).

[12] A. M. Lindenberg, S. L. Johnson, and D. A. Reis, Annu. Rev. Mater. Res. 47, 425 (2017).

[13] M. Mitrano, A. Cantaluppi, D. Nicoletti, S. Kaiser, A. Perucchi, S. Lupi, P. Di Pietro, D. Pontiroli, M. Riccò, S. R. Clark, D. Jaksch, and A. Cavalleri, Nature (London) 530, 461 (2016).

[14] D. Rudolf, C. La-O-Vorakiat, M. Battiato, R. Adam, J. M. Shaw, E. Turgut, P. Maldonado, S. Mathias, P. Grychtol, H. T. Nembach, T. J. Silva, M. Aeschlimann, H. C. Kapteyn, M. M. Murnane, C. M. Schneider, and P. M. Oppeneer, Nat. Commun. 3, 1037 (2012). 
[15] I. Razdolski, A. Alekhin, N. Ilin, J. P. Meyburg, V. Roddatis, D. Diesing, U. Bovensiepen, and A. Melnikov, Nat. Commun. 8, 15007 (2017).

[16] T. Seifert, S. Jaiswal, U. Martens, J. Hannegan, L. Braun, P. Maldonado, F. Freimuth, A. Kronenberg, J. Henrizi, I. Radu, E. Beaurepaire, Y. Mokrousov, P. M. Oppeneer, M. Jourdan, G. Jakob, D. Turchinovich, L. M. Hayden, M. Wolf, M. Münzenberg, M. Kläui, and T. Kampfrath, Nat. Photon. 10, 483 (2016)

[17] D. Bossini, K. Konishi, S. Toyoda, T. Arima, J. Yumoto, and M. Kuwata-Gonokami, Nat. Phys. 14, 370 (2018).

[18] M. I. Kaganov, I. M. Lifshitz, and L. V. Tanatarov, Sov. Phys. JETP 4, 173 (1957)

[19] S. I. Anisimov, B. L. Kapeliovich, and T. L. Perel'man, Sov. Phys. JETP 39, 375 (1974)

[20] P. B. Allen, Phys. Rev. Lett. 59, 1460 (1987).

[21] J. Simoni and J. Daligault, Phys. Rev. Lett. 122, 205001 (2019).

[22] L. Waldecker, R. Bertoni, R. Ernstorfer, and J. Vorberger, Phys. Rev. X 6, 021003 (2016).

[23] L. Waldecker, T. Vasileiadis, R. Bertoni, R. Ernstorfer, T. Zier, F. H. Valencia, M. E. Garcia, and E. S. Zijlstra, Phys. Rev. B 95, 054302 (2017).

[24] H. Aoki, N. Tsuji, M. Eckstein, M. Kollar, T. Oka, and P. Werner, Rev. Mod. Phys. 86, 779 (2014).

[25] V. V. Baranov and V. V. Kabanov, Phys. Rev. B 89, 125102 (2014)

[26] E. Carpene, Phys. Rev. B 74, 024301 (2006).

[27] B. Rethfeld, A. Kaiser, M. Vicanek, and G. Simon, Phys. Rev. B 65, 214303 (2002).

[28] Z. Lin, L. V. Zhigilei, and V. Celli, Phys. Rev. B 77, 075133 (2008)

[29] M. Bauer, A. Marienfeld, and M. Aeschlimann, Prog. Surf. Sci. 90, 319 (2015).

[30] Y. Murakami, P. Werner, N. Tsuji, and H. Aoki, Phys. Rev. B 91, 045128 (2015).

[31] S. Ono, Phys. Rev. B 97, 054310 (2018).

[32] O. Abdurazakov, D. Nevola, A. Rustagi, J. K. Freericks, D. B. Dougherty, and A. F. Kemper, Phys. Rev. B 98, 245110 (2018).

[33] I. Klett and B. Rethfeld, Phys. Rev. B 98, 144306 (2018).

[34] A. Tamm, M. Caro, A. Caro, G. Samolyuk, M. Klintenberg, and A. A. Correa, Phys. Rev. Lett. 120, 185501 (2018).

[35] A. H. Reid, X. Shen, P. Maldonado, T. Chase, E. Jal, P. W. Granitzka, K. Carva, R. K. Li, J. Li, L. Wu, T. Vecchione, T. Liu, Z. Chen, D. J. Higley, N. Hartmann, R. Coffee, J. Wu, G. L. Dakovski, W. F. Schlotter, H. Ohldag et al., Nat. Commun. 9, 388 (2018).

[36] P. Maldonado, K. Carva, M. Flammer, and P. M. Oppeneer, Phys. Rev. B 96, 174439 (2017).

[37] S. T. Weber and B. Rethfeld, Phys. Rev. B 99, 174314 (2019).

[38] S. Sadasivam, M. K. Y. Chan, and P. Darancet, Phys. Rev. Lett. 119, 136602 (2017).

[39] P. Maldonado, T. Chase, A. H. Reid, X. Shen, R. K. Li, K. Carva, T. Payer, M. Horn von Hoegen, K. Sokolowski-Tinten, X. J. Wang, P. M. Oppeneer, and H. A. Dürr, Phys. Rev. B 101, 100302(R) (2020).

[40] S. Ono and T. Suemoto, Phys. Rev. B 102, 024308 (2020).
[41] I. Gierz, F. Calegari, S. Aeschlimann, M. Chávez Cervantes, C. Cacho, R. T. Chapman, E. Springate, S. Link, U. Starke, C. R. Ast, and A. Cavalleri, Phys. Rev. Lett. 115, 086803 (2015).

[42] G. Rohde, A. Stange, A. Müller, M. Behrendt, L.-P. Oloff, K. Hanff, T. J. Albert, P. Hein, K. Rossnagel, and M. Bauer, Phys. Rev. Lett. 121, 256401 (2018).

[43] A. F. Kemper, O. Abdurazakov, and J. K. Freericks, Phys. Rev. X 8, 041009 (2018).

[44] P. Tengdin, W. You, C. Chen, X. Shi, D. Zusin, Y. Zhang, C. Gentry, A. Blonsky, M. Keller, P. M. Oppeneer, H. C. Kapteyn, Z. Tao, and M. M. Murnane, Sci. Adv. 4, eaap9744 (2018).

[45] T. Konstantinova, J. D. Rameau, A. H. Reid, O. Abdurazakov, L. Wu, R. Li, X. Shen, G. Gu, Y. Huang, L. Rettig, I. Avigo, M. Ligges, J. K. Freericks, A. F. Kemper, H. A. Dür, U. Bovensiepen, P. D. Johnson, X. Wang, and Y. Zhu, Sci. Adv. 4, eaap7427 (2018).

[46] M. Bernardi, Eur. Phys. J. B 89, 239 (2016).

[47] A. Togo, L. Chaput, and I. Tanaka, Phys. Rev. B 91, 094306 (2015).

[48] F. Murphy-Armando, D. Murray, I. Savić, M. Trigo, D. Reis, and S. Fahy, arXiv:1911.12145.

[49] G. P. Srivastava, The Physics of Phonons (Taylor \& Francis, New York, 1990).

[50] P. Maldonado and Y. O. Kvashnin, Phys. Rev. B 100, 014430 (2019).

[51] M. Schütt, P. P. Orth, A. Levchenko, and R. M. Fernandes, Phys. Rev. B 97, 035135 (2018).

[52] J. Kimling, G.-M. Choi, J. T. Brangham, T. Matalla-Wagner, T. Huebner, T. Kuschel, F. Yang, and D. G. Cahill, Phys. Rev. Lett. 118, 057201 (2017).

[53] J. Barker and G. E. W. Bauer, Phys. Rev. B 100, 140401(R) (2019).

[54] M. Beens, M. L. M. Lalieu, A. J. M. Deenen, R. A. Duine, and B. Koopmans, Phys. Rev. B 100, 220409(R) (2019).

[55] A. Lyberatos and G. J. Parker, Jpn. J. Appl. Phys. 58, 045002 (2019).

[56] M. Obergfell and J. Demsar, Phys. Rev. Lett. 124, 037401 (2020).

[57] H. Jang, J. Kimling, and D. G. Cahill, Phys. Rev. B 101, 064304 (2020).

[58] M. Trigo, M. Fuchs, J. Chen, M. P. Jiang, M. Cammarata, S. Fahy, D. M. Fritz, K. Gaffney, S. Ghimire, A. Higginbotham, S. L. Johnson, M. E. Kozina, J. Larsson, A. M. Lemke, H. Lindenberg, G. Ndabashimiye, F. Quirin, K. SokolowskiTinten, C. Uher, G. Wang et al., Nat. Phys. 9, 790 (2013).

[59] X. Gonze, B. Amadon, P. M. Anglade, J. M. Beuken, F. Bottin, P. Boulanger, F. Bruneval, D. Caliste, R. Caracas, M. Cote, T. Deutsch, L. Genovese, P. Ghosez, M. Giantomassi, S. Goedecker, D. R. Hamann, P. Hermet, F. Jollet, G. Jomard, S. Leroux, M. Mancini et al., Comput. Phys. Commun. 180, 2582 (2009).

[60] We compared our values with http://www.faculty.virginia.edu/ CompMat/electron-phonon-coupling.

[61] X. Tang, C. W. Li, and B. Fultz, Phys. Rev. B 82, 184301 (2010)

[62] G. Kresse and J. Furthmüller, Phys. Rev. B 54, 11169 (1996). 